\title{
A preliminary exploration of the application of self-compassion within the context of sport injury
}

Zenzi Huysmans

Follow this and additional works at: https://researchrepository.wvu.edu/etd

\section{Recommended Citation}

Huysmans, Zenzi, "A preliminary exploration of the application of self-compassion within the context of sport injury" (2016). Graduate Theses, Dissertations, and Problem Reports. 5848.

https://researchrepository.wvu.edu/etd/5848

This Thesis is protected by copyright and/or related rights. It has been brought to you by the The Research Repository @ WVU with permission from the rights-holder(s). You are free to use this Thesis in any way that is permitted by the copyright and related rights legislation that applies to your use. For other uses you must obtain permission from the rights-holder(s) directly, unless additional rights are indicated by a Creative Commons license in the record and/ or on the work itself. This Thesis has been accepted for inclusion in WVU Graduate Theses, Dissertations, and Problem Reports collection by an authorized administrator of The Research Repository @ WVU. For more information, please contact researchrepository@mail.wvu.edu. 
A preliminary exploration of the application of self-compassion within the context of sport injury

\section{Zenzi Huysmans}

Thesis submitted to the College of Physical Activity and Sport Sciences at West Virginia University in partial fulfillment of the requirements for the degree of Master of Science in Sport and Exercise Psychology

Damien Clement, Ph.D., Chair

Samuel Zizzi, Ph.D.

Monica Leppma, Ph.D.

Department of Sport Sciences

Morgantown, WV, USA

2016

Keywords: stress, coping, injury, self-compassion, collegiate athletes

Copyright 2016 Zenzi Huysmans 


\begin{abstract}
A preliminary exploration of the application of self-compassion within the context of sport injury

Zenzi Huysmans
\end{abstract}

Self-compassion is a useful coping tool to enhance adaptive coping, promote well-being, and reduce anxiety in the face of stress (Neff, 2003a; Allen \& Leary, 2010). Studies exploring the role of self-compassion within the context of sport indicate the utility of self-compassion for female athletes in coping with negative events in sport (Mosewich et al., 2013), promoting positivity and responsibility (Ferguson et al., 2014), and reducing negative self-evaluative thoughts (Mosewich et al, 2011). Within the context of Williams and Andersen's (1998) stressinjury framework and the moderating effect of coping resources on injury susceptibility, selfcompassion was newly explored. Specifically, this study aimed to determine the role of selfcompassion as a coping resource and a tool to cognitively reframe stressful sport and life experiences in collegiate athletes. The subsequent impact on frequency and severity of injury was also explored. Participants were collegiate athletes $(n=117)$ who participated in NCAA Division II sports at one university in the Mid-Atlantic region of the United States. A prospective study design was used to evaluate the associations between baseline measures of stress, anxiety and coping and frequency or severity of injury during the competitive season. Bi-variate analyses and multiple linear regression modeling were used to analyze the results. Findings indicated that self-compassion may buffer the experience of somatic anxiety $(r s=-.436, p<.001)$ and worry $(r s=-.351, p<.001)$ in collegiate athletes and reduce the engagement of avoidance-focused coping strategies $(r s=-.362, p<.001)$. However, several of the findings were in contrast with self-compassion research in the general population, which included a negative association between self-compassion and emotion-focused coping ( $r s=-.259, p<.001)$. There were no significant findings related to the role of self-compassion in injury risk reduction. Findings that focused specifically on the traditional components of Williams and Andersen's stress-injury model were also mixed. A negative association was found between positive life stress and frequency of injury $(r s=-.257, p<.05)$, suggesting that certain positive experiences may be reduce injury risk. Additionally, negative life stress was negatively associated with emotionfocused $(r s=-.242, p<.05)$ and avoidance-focused coping $(r s=-.249, p<.05)$. However, there were no significant findings for negative life stress and injury. In summary, findings confirmed the role of several psychological factors in the stress-injury framework and indicated that there may be some use for self-compassion within the context of sport. There is a need to further assess levels of self-compassion in collegiate athletes as well as the role of self-compassion as a focus for interventions to lower stress, anxiety and injury susceptibility. 


\section{Acknowledgements}

This thesis milestone would not have been possible without the help of all the wonderful people I have surrounding me. My advisor and chair of my thesis committee, Dr. Clement, has been a thoughtful and constructive force throughout the process. Not only did he read and edit countless drafts of my work, but he was also a source of encouragement and positivity when I encountered set-backs. Self-compassion is not his area of expertise yet he allowed me to pursue my passion and was willing to learn a great deal about this area of research along the way. Dr. Zizzi was the second member of my thesis committee. He was the statistics guru for this project and successfully helped me push myself to the limits of my statistics knowledge to elevate the quality of my work. Dr. Leppma was the self-compassion expert on my committee. Self-compassion is being newly applied to the sporting context so we both enjoyed exploring the applicability of self-compassion for athletes and its promise as a tool for health and well-being. True to her research, she was a balanced and compassionate member of my committee.

My colleagues and friends in the WVU Sport and Exercise Psychology program were phenomenal sounding boards for research ideas and were also my closest sources of social support with my family so far away. I have been lucky to find some good friends in this program who have supported and loved me unconditionally.

Above all, my family (my mum, sister and significant other) has been the rock that kept me grounded. If I ever doubted myself, they were there to remind me of my strength and their love, and inspired me to work towards my dreams.

Thank you!! 
Table of Contents

$\begin{array}{ll}\text { Introduction } & 1\end{array}$

$\begin{array}{ll}\text { Methodology } & 7\end{array}$

$\begin{array}{ll}\text { Participants } & 7\end{array}$

$\begin{array}{ll}\text { Measures } & 8\end{array}$

$\begin{array}{ll}\text { Procedure } & 11\end{array}$

$\begin{array}{ll}\text { Data Analysis } & 12\end{array}$

$\begin{array}{ll}\text { Results } & 13\end{array}$

$\begin{array}{ll}\text { Discussion } & 16\end{array}$

$\begin{array}{ll}\text { Study Overview } & 16\end{array}$

Williams and Andersen's Stress-Injury Model 17

Stress, Coping, Injury and Self-Compassion 26

Future Directions 31

$\begin{array}{ll}\text { Limitations } & 34\end{array}$

Conclusion $\quad 36$

$\begin{array}{ll}\text { References } & 38\end{array}$

$\begin{array}{ll}\text { Appendix A- Tables } & 47\end{array}$

Table 1: Sample Characteristics $\quad 47$

Table 2: Descriptive Statistics $\quad 48$

Table 3: Descriptive Injury Data from Competitive Season 49

Table 4: Associations between Pre-Season Study Variables $\quad 50$

Appendix B- Extended Literature Review 51

Appendix C- Demographic Information 95

Appendix D: Life Events Survey for Collegiate Athletes (LESCA) 96 
$\begin{array}{ll}\text { Appendix E: Sport Anxiety Scale (SAS) } & 102\end{array}$

Appendix F: Self-Compassion Scale- Short Form (SCS-SF) 103

$\begin{array}{ll}\text { Appendix G: Brief COPE } & 104\end{array}$

Appendix H: Athletic Coping Skills Inventory (ACSI-28) 106

Appendix I: Order of Survey Packets 109 
A Preliminary Exploration of the Application of Self-Compassion within the Context of Sport

$$
\text { Injury }
$$

Risk of injury is an intrinsic component of playing recreational and competitive sport and is largely accepted as being part of the sporting culture (Conn, Annest, \& Gilchrist, 2003; Uitenbroek, 1996; Nixon, 1993). Injury rates vary depending on gender (Conn, Annest, \& Gilchrist, 2003), age-group (Finch \& Cassell, 2006) as well as participation in team ball sports versus individual sports (Finch \& Cassell, 2006). It is estimated that $22 \%$ of emergency room visits for individuals aged 5 to 24 years old are attributed to sport-related injuries, which are more likely to be to the head, upper and lower extremities when compared to non-sport related injuries (Burt \& Overpeck, 2001). Unfortunately, sport injury can cause lengthy absences from play (Finch \& Cassell, 2006) as well as psychological distress in the form of decreases in selfworth and self-confidence, increases in mood disturbance (Wiese-Bjornstal et al., 1998) and in some cases clinical issues (Nippert \& Smith, 2008; Brewer, 2001). The development of comprehensive injury prevention initiatives that are aimed at modifiable risk factors for injury are therefore warranted (Hootman, Dick, \& Agel, 2007).

Psychological factors play a significant role in risk for injury (Williams, 2001; Junge, 2000). Theoretical and empirical evidence suggests that stress and coping factors are linked to athletic injury (Blackwell \& McCullagh, 1990; Shamus, Kelleher \& Foran, 2001) and to date, Andersen and Williams' (1988) stress-injury model is the most influential multivariable approach to predicting acute athletic injury (Johnson, 2007). Studies across a wide range of sports and athletic populations, as well as qualitative and intervention studies, provide support for the stress-injury association (Johnson, 2007). Specifically, high life stress (especially negative life stress) has been associated with increased susceptibility to injury e.g. 6-12\% in 
gymnasts (Petrie, 1992) while further studies indicate that coping resources and social support in combination with life stress further exacerbates the stress injury relationship (Johnson, 2007). Smith, Smoll and Ptacek (1990) found that 22-30\% of time loss due to injury was predicted by high negative life stress, low social support and low coping resources. Although numerous studies support the stress-injury association, further consolidating studies are necessary to shed light on the role of moderating variables in Williams and Andersen's model (Maddison \& Prapavessis, 2005). Additionally, there is a need to explore the stress-injury association in a wider range of athletic populations (Petrie, 1993). That is, although stress may be experienced by the majority of athletes regardless of the type of sport or level of competition (Mellalieu et al, 2009; Hanton, Fletcher \& Coughlan, 2005; Giacobbi, Foore \& Weinberg, 2004; Holt \& Hogg, 2002; Noblet \& Gifford, 2002; Gould, 1993; Passer, 1981), collegiate athletes are particularly susceptible to elevated levels of stress due to the presence of unique stressors related to sport. This may include isolation from the general student body, lack of control over their athletic and academic experience, performance pressures, overtraining and other sport-specific issues (Etzel, 2009; Kaiseler, Polman \& Nicholls, 2009; Etzel et al., 2006; Etzel \& Watson, 2007; Pinkerton et al., 1989). Additionally, they also face the more widespread stressors of trying to balance the academic and psychosocial domains of their college experience (Etzel, 2009; Etzel et al., 2006). Explorations into stress and injury reduction strategies in collegiate athletes are therefore called for.

According to Williams and Andersen's (1998) conceptualization of the stress-injury association, interventions for injury reduction are two-pronged: either they address cognitive appraisal of stress or they address the physiological aspects of the stress response in order to reduce stress reactivity. Cognitive appraisal is defined as the way an individual evaluates a threat 
and their ability to respond to that threat (Lazarus \& Folkman, 1984). The inclusion of appraisal in Lazarus and Folkman's (1984) model dictates that the focus of coping should not be on the stressor but rather on the individual's appraisal of the stressor. This is because an individual's ability to cope with a situation that is perceived to be stressful will be largely affected by their perception of their capacity to respond to the stress (Carver, Scheier, \& Weintraub, 1989;

Lazarus, 1966; Lazarus \& Folkman, 1984). This has been supported in more recent studies. Hanton, Wagstaff, and Fletcher (2012) and Neil et al. (2011) studied the role of cognitive appraisal and stress in elite athletes. Findings indicated that cognitive re-appraisal of either the stressor itself or the athlete's emotional response to the stressor resulted in more adaptive responses to the stressful situation e.g. increased concentration and effort (Neil et al., 2011) or taking action and getting on with it (Hanton et al., 2012).

These findings are consequential because they highlight the importance of the process of cognitive appraisal of, and emotional response to, stressors. Furthermore, they imply that interventions or psychological variables that intervene at the appraisal stage of the stress response could moderate the impact that stressors have on elite and collegiate athletes. That is, in situations where the stressor cannot be changed e.g. those intrinsic to sport such as training load, the stress response can be reduced by focusing on minimizing threat appraisals and instead facilitating challenge appraisals. This could have implications on injury risk reduction strategies. Therefore, further exploration of psychological factors and stress reduction techniques that target appraisal of stress among collegiate athletes is warranted.

\section{Self-Compassion, Cognitive Appraisal of Stress and Injury Risk Reduction}

This study is proposing that self-compassion may be a key psychological variable to explore within the context of cognitive appraisal of stress in sport and the stress-injury 
relationship. Self-compassion is composed of three distinct concepts: self-kindness in times of perceived inadequacy, common humanity, and balanced awareness of emotions (Neff, 2003a; Neff, 2003b). Self-compassion enhances adaptive coping, positive psychological functioning and well-being, and reduces anxiety in the face of stress (Neely et al., 2009; Neff, 2003a; Neff, Rude \& Kirkpatrick, 2007, Neff, 2003b; Neff, Kirkpatrick \& Rude, 2007; Allen \& Leary, 2010). Furthermore, it contributes to the appraisal process by changing the experience of self-evaluative appraisals (Neff, 2003a; Neff et al., 2007a; Mosewich et al., 2011). It is characterized as a form of coping that uses positive cognitive reframing (Allen \& Leary, 2010). This may allow selfcompassion to serve as a buffer when athletes encounter stressful experiences.

Importantly, self-compassion is a form of emotional approach coping (Neff, 2003a). Emotion-focused coping involves managing the emotional distress associated with the situation (Folkman \& Lazarus, 1980; Folkman \& Lazarus, 1985). This allows an individual to change the way he/she thinks about a stressor and accept responsibility for the role they play in the unfolding of a negative event while maintaining balanced awareness of emotions (Allen \& Leary, 2010; Leary et al., 2007; Neff, Hsieh \& Dejitterat, 2005). That is, self-compassion promotes proactive behaviors as opposed to passivity. Specifically, self-compassion is positively associated with the emotion-focused strategies of growth and acceptance and negatively associated with the avoidance-coping strategies of denial and mental disengagement (Neff, Hsieh \& Dejitterat, 2005). Avoidant coping involves psychologically or behaviorally avoiding the stressful situation through mental states such as withdrawal or activities such as alcohol (Carver et al., 1989; Herman-Stabl, 1995). Athletes higher in avoidance-focused coping have been found to display a stronger stress-injury relationship (Maddison \& Prapavessis, 2005). Focusing on emotions may in fact allow the individual to re-appraise the stressor and come to a healthier 
attribution of the event (Stanton et al., 2000). Overall, these studies suggest that self-compassion has an impact on coping and the stress response through its effects on cognitive appraisal of stress. That is, within sport, self-compassion may help the athlete positively reframe an experience of stress, and therefore possibly reduce the stress response and its associated increase in physiological arousal and attentional disruptions. This is pertinent to Williams and Andersen's suggestion that intervention to reduce the stress-injury relationship should focus on psychological factors or stress management techniques that focus on cognitive appraisal of stress.

Within the sport domain, empirical evidence supports the value of self-compassion for female athletes. Mosewich et al. (2011) investigated the applicability of self-compassion within the context of self-evaluative thoughts and behaviors and self-conscious emotions in high-school and club-level female athletes $(n=151)$. Self-compassion was found to be negatively correlated with shame-proneness $(r=-.32, p<.01)$, fear of failure $(r=-.48, p<.01)$, and fear of negative evaluation $(r=-.48, p<.01)$. It was concluded that self-compassion may help female athletes respond more adaptively to negative events of failure or disappointment, which may cause stress, by accepting their responsibility in causing the event without harsh self-criticism (Mosewich et al., 2011). Another study explored the association between self-compassion and eudemonic well-being in high-school and varsity female athletes $(n=83)$ (Ferguson et al., 2014). It was found that self-compassion was positively associated with eudemonic well-being $(r=.76, p<$ .01) while mediation analyses indicated that self-compassion may promote well-being through active engagement, taking initiative and responsibility. Intervention studies also support the applicability of self-compassion in female athletes. Mosewich et al. (2013) explored the application of a self-compassion intervention on negative cognitive states in female varsity 
athletes $(n=60)$ in Western Canada. The purpose of the study was to teach athletes to apply elements of self-compassion when faced with negative events in their sport. The intervention consisted of several self-compassion writing modules and a psychoeducation session to be completed over a 7-day period. Findings indicated that the intervention was effective in increasing self-compassion and helping the athletes manage their concern over mistakes, rumination and self-criticism, with a moderate-to-large effect size (Wilks's $\Lambda=.566, F(8,42)=$ 4.03, $p<.01, \eta 2=.43)$ (Mosewich et al., 2013).

\section{Current Study}

Overall, these studies indicate that self-compassion may be a potential resource in the stress experience of female athletes by promoting well-being, managing concern over mistakes and self-criticism and responding adaptively to negative events (Mosewich et al., 2011; Mosewich et al, 2013; Reis et al., 2015). However, self-compassion has yet to be studied within the context of antecedents to injury and as a possible moderator of the stress-injury association for either male or female athletes. This study predicted that self-compassion may potentially serve as a coping resource and a way to cognitively re-appraise a stressful experience, and therefore serve as a moderator of the relationship between stress and athletic injury in collegiate athletes. Specifically, this study proposed that within the sport psychology context, appraisal of stress and the ability to cope more effectively with stress will be the key concepts that connect self-compassion, stress and injury within the framework of Williams and Andersen's (1998) stress-injury model. The following hypotheses were generated: Hypothesis 1: life stress would have a small positive association with frequency and severity of injury. Hypothesis 2: coping resources would show a negative association with frequency and severity of injury. Hypothesis 3: self-compassion would have a small negative association with scores on the life stress 
measure, a small positive association with emotion-focused coping and a small negative association with avoidance-focused coping. Hypothesis 4: self-compassion would be negatively associated with competitive sport anxiety. Hypothesis 5: higher levels of coping would be associated with lower levels of life stress. Hypothesis 6: self-compassion and competitive sport anxiety would combine with coping resources and life stress as significant predictors of frequency and severity of injury.

\section{Methodology}

\section{Participants}

Participants in this study were collegiate athletes from one university in the Mid-Atlantic region of the United States. One hundred and seventeen ( $n=117)$ athletes aged 18-26 who participated in NCAA Division II sports (men's team was football; women's teams were volleyball and soccer) between August 20, 2015, and November 30, 2015 were included in the study after eleven participants were excluded for incorrectly following survey instructions or for quitting their team during the season. Of the 117 participants, approximately one-third were female $(n=$ $36,30.8 \%)$ and two-thirds was male $(n=81,69.2 \%)$. Table 1 displays participant characteristics (See Appendix A). The average participant age was $19.50(S D=1.57)$ years, while $41.9 \%(n=$ $49)$ of the participants were White or Caucasian, and 40.2\% $(n=47)$ were Black or African American. Over one-third of the participants were freshmen $(n=42,35.9 \%), 25.6 \%(n=30)$ were sophomores, and 24.8\% $(n=29)$ were juniors. Football players comprised the majority of the study participants $(n=81,69.2 \%)$, followed by women's soccer $(n=23,19.7 \%)$ and women's volleyball $(n=13,11.1 \%)$. Approximately $35.0 \%(n=41)$ of the athletes were starters on their team, and 40.2\% $(n=47)$ were second string. Twenty one percent $(n=25)$ reported 
suffering at least one injury in the 12 months preceding the study of which $53.1 \%(n=17)$ were major injuries.

\section{Measures}

Demographic information. Participants completed a demographic sheet assessing age, gender, ethnicity, year in school, playing status, years playing and injury history (See Appendix C). Life Stress. The Life Events Survey for Collegiate Athletes (LESCA; Petrie, 1992) was administered to measure which life event stressors (including sport-specific stressors) athletes experienced during the preceding twelve months. The LESCA is a 69 item survey that assesses athletes' response to an event on an eight-point Likert scale $(-4=$ extremely negative to $+4=$ extremely positive). Scores on each item are summed to give three different life event scores: negative (scores range from 0 to -276 ), positive (scores range from 0 to +276 ) and total (sum of positive and negative scores). This instrument was developed using intercollegiate athletes and has been shown to have excellent psychometric properties including moderate criterion-related validity between positive and negative life stress scores $(r=.55, p<0.05)$ (Petrie, 1993), high testretest reliability (.76-.84) (Petrie, 1993), and significant construct validity (Petrie, 1992; Petrie, 1993; Andersen \& Williams, 1999) (See Appendix D).

Competitive Trait Anxiety. The Sport Anxiety Scale (SAS; Smith, Smoll \& Schutz, 1990b) is a 21-item measure of trait anxiety and includes three interrelated scales (somatic anxiety, worry (cognitive anxiety), and concentration disruption (cognitive anxiety)). Each item is scored on a four-point Likert scale $(1=$ not at all to $4=$ very much). Separate scores are obtained for each scale by summing item ratings: somatic anxiety (scores range from 9 to 36), worry (scores range from 7 to 28) and concentration disruption (scores range from 5 to 20). In two samples of athletes, Cronbach's alpha has been found to range between .74 and .92 for each scale. In a 
sample of college-aged athletes, test-re-test reliability exceeded 0.85 on all scales (Petrie, 1993;

Smith et al., 1990b) (See Appendix E).

Self-Compassion. Participants were asked to complete the 12-item Self-Compassion Scale-Short Form (SCS-SF; Raes et al., 2011). The SCS-SF assesses three different aspects of selfcompassion: self-kindness versus self-judgment; common humanity versus isolation; and mindfulness versus over-identification. A five-point Likert scale is used to rate each item on the subscales ( $1=$ almost never to $5=$ =almost always). An overall self-compassion score (scores range from 6 to 30) is obtained by reverse coding the negative items and summing up the mean scores on the six subscales. Scale validation research for the SCS-SF indicates that within German and English samples, all six subscales are highly inter-correlated and the SCS-SF demonstrates good internal consistency (Cronbach's alpha $\geq 0.86)$ (Neff, 2003a; Neff et al., 2005). The SCS-SF has also been found to be nearly perfectly correlated to the long form SCS ( $r>.97)$ (Raes et al., 2011) (See Appendix F).

Coping Style. The Brief COPE inventory (Brief COPE; Carver, 1997) is a 28-item survey that measures three conceptually distinct forms of coping: problem-focused coping, emotion-focused coping and dysfunctional coping. There are fourteen subscales (active coping, planning, positive reframing, acceptance, humor, turning to religion, using emotional support, using instrumental support, self-distraction, denial, venting, substance use, behavioral disengagement, and selfblame) with two items on each subscale. Each item is scored on a four-point Likert scale (1= I haven't been doing this at all to 4= I have been doing this a lot). Total scores are calculated for each of the fourteen subscales by summing the two items of the subscale. The subscales can be collapsed into different categories of coping but this is left to the user's discretion. Reliability and validity statistics have consistently been found to be adequate in various non-sport 
populations; Cronbach's alpha ranges from 0.5 to 0.9 (Carver, 1997; Cooper, Katona \& Livingston, 2008; Yusoff, Low \& Yip, 2010). Carver suggests that subscales can be selected individually according to preference for data analysis (Carver, 1997) (See Appendix G). Subsequently, an emotion-focused coping scale was derived by summing values from the positive reframing and acceptance subscales while an avoidance-focused coping scale was derived by summing values from the denial, self-distraction, and behavioral disengagement subscales. These sub-scales were chosen given that self-compassion has been found to be positively associated with the emotion-focused strategies of growth and acceptance and negatively associated with the avoidance-coping strategies of denial and mental disengagement (Neff, Hsieh \& Dejitterat, 2005).

Coping. The Athletic Coping Skills Inventory (ACSI-28; Smith et al., 1995) is a 28-item survey that contains seven sport-specific subscales assessing coping: coping with adversity, peaking under pressure, goal setting and mental preparation, concentration, freedom of worry, confidence and achievement motivation, and coachability. A four-point Likert scale is used $(0=$ almost never to $3=$ almost always). Scores from each subscale are summed to yield a Personal Coping Resources score (scores range from 0 to 84 ). Internal consistency and test-re-test reliability coefficients have been found to be 0.86 and 0.87 respectively (Smith et al., 1995) (See Appendix $\mathrm{H})$.

Injury Recording. Injuries were defined as all types of injuries that occurred in connection with sport participation and resulted in at least one day of missed or modified (i.e. reduced participation, strapping etc.) practice or competition (Ford, Eklund \& Gordon, 2000; Andersen \& Williams, 1999; Lysens, Weerdt, \& Nieuwboer, 1991), and/or required treatment (Maddison \& Prapavessis, 2005). Injury records were collected by a certified athletic trainer throughout the 
course of the fall 2015 competitive season using the Athletic Trainer System (ATS). The severity of injuries were conceptualized as a continuous variable of "time loss" due to injury i.e. the length of time in days that the athlete did not complete a full practice, which included modified practices, due to injury. If the athlete sustained several injuries, time loss was computed by adding the amount of time missed for each of the separate injuries to create a total time loss score. A separate time loss variable for severity was computed to also include the number of days in which the athletes were cleared for full play by the certified athletic trainer but were told to modify practice if needed based on their own judgment. The frequency of separate injuries incurred that resulted in at least one day missed (or modified) of practice or competition were also recorded (Andersen \& Williams, 1999; Ford, Eklund \& Gordon, 2000). Frequency of injury was kept as a continuous variable.

\section{Procedure}

Study procedures were approved by the Institutional Review Board prior to the collection of data. Convenience sampling was used to select athletic teams at a Mid-Atlantic university and approval was gained by the principal investigator from coaches and certified athletic trainers of the respective teams. Subsequently, a meeting was set up with each team where a questionnaire packet was administered and collected by a research assistant before the start of each team's competitive season. Questionnaire packets were ordered in 3 different ways in order to assess for ordering effects (see Appendix I). All athletes present at the meeting were invited to participate in the study and were asked to complete a signed consent form after the purposes of the study were explained. Over the course of the competitive season injuries were logged and tracked by the athletic trainers using the ATS. The ATS database contains detailed information across 
numerous domains of the athletes' lives outside of sport but also tracks injury information such as date, type and location of injuries. Data was entered into SPSS and cleaned.

\section{Data Analysis}

\section{Missing Data and Evaluation of Assumptions}

Data cleaning and evaluation of assumptions included: 1) checking for outliers i.e. ascending and descending data as well as eyeballing, 2) checking ranges of data to ensure that they fell within the parameters of each measure, 3) computing total and sub-scale scores for each measure, and 4) running reliability descriptives for each measure. If an athlete skipped questions on a measure or did not complete certain surveys, they were still included in the study but those surveys with missing data were not included in the analyses. After preliminary normality tests were conducted, the predictor data appeared to violate the assumption of normality. That is, z-scores and histograms indicated distinct skewing. Data from each measure was subsequently transformed using the appropriate transformation (reflection, logarithm, reciprocal) dependent on the distribution of the data. Additionally, it was decided that Spearman's Rho would be more appropriate for the bivariate analyses as opposed to Pearson's correlation.

An a priori power analysis was conducted using the statistical program G-Power to identify an adequate sample size of 80 , assuming small effect size. Statistical analyses consisted of descriptive statistics and bivariate analyses (i.e. Spearman's Rho correlations) to examine associations between participant characteristics and predictor variables as well as correlations amongst the predictor variables (self-compassion, life stress, coping, and competitive trait anxiety). Subsequent to implementing the appropriate data transformations based on distribution issues, generalized linear regression modeling was used with a standard entry approach to assess the amount of variance in injury frequency or severity that could be accounted for by the pre- 
season predictor variables. A standard entry approach was used in order to assess the unique contributions of each predictor as compared to the other variables in the model. Additionally, given that the Brief COPE and Self-Compassion Scale had not been used in previous injury research, there was no basis for comparison in terms of predicting which predictor variable would create the strongest regression equation.

\section{Results}

\section{Descriptive Data}

Predictor Variables. Table 2 (See Appendix A) displays complete descriptive data for the preseason study variables. The ACSI indicated that average sport-specific Personal Resource Coping Score was $53.35(S D=10.54$; range, $32-78)$ with Coachability $(M=9.54$; $S D=2.02$; range, 4-12) as the highest average subscale and Freedom from Worry $(M=6.61 ; S D=2.62$; range, $0-12$ ) as the lowest average subscale. The average scores for the Brief COPE were highest for Active coping $(M=5.34 ; S D=1.85$; range, $2-8)$ and lowest for Substance use $(M=2.42$; $S D=1.10$; range, 2-7). Additionally, the average emotion-focused coping score was 8.85 $(S D=3.42$; range, $4-16)$ and the average avoidance-focused coping score was $10.26(S D=3.39$; range, 6-20). The SAS indicated that Somatic Anxiety was on average 16.13 (SD=5.61; range, 9-34) and Worry was on average 14.25 ( $S D=5.11$; range, 7-28). The average Self-Compassion score was 18.84 ( $S D=3.56$; range, 6-28.5). On the LESCA measure, negative life stress was on average $-15.17(S D=18.06$; range, $-122-0)$ while positive life stress was on average $17.46(S D=$ 15.87; range, 0-74). There were no significant gender differences across pre-season measures except for in SAS Worry $(t(115)=-2.57, p<0.05, d=0.48)$ where women scored higher $(M=16.00)$ in SAS Worry than men $(M=13.47)$ with small effect size, and the ACSI Personal 
Resources Coping Score $(t(110)=2.59, p<0.05, d=0.49)$ where men scored higher $(M=55.18)$ than women $(M=49.47)$ in sport-specific coping resources with small effect size.

Injury Outcome Data. Over the course of the 2015 competitive season $51.3 \%(n=60)$ of the athletes did not incur an injury, while $27.4 \%(n=32)$ sustained one injury, and $21.4 \%(n=25)$ sustained two or more injuries. Table 3 (See Appendix A) displays descriptive data for injuries across the season. Overall there were 91 separate injuries across the three sports: ankle/foot $(n=15,16.5 \%)$, knee injuries $(n=11,12.1 \%)$, shoulder injuries $(n=19,20.8 \%)$, concussions $(n=5$, $5.5 \%)$, back injuries $(n=3,3.3 \%)$, and uncategorized injuries $(n=38,41.8 \%)$. Injury rate was similar in football and volleyball with $54.3 \%(n=44)$ of the football players sustaining at least one injury compared to $53.8 \%(n=7)$ of the volleyball players. In soccer, injury rate was $26.1 \%$ $(n=6)$. Of the male athletes, over half $(54.3 \%, n=44)$ sustained at least one injury while just over a third $(36.1 \%, n=13)$ of the female athletes sustained at least one injury $(t(85.6)=-2.81$, $p<0.05, d=0.61)$. There was a significant difference in frequency of injury between athletes with a recent history of injury (12 preceding months) and those without a history of injury $(t(115)=-$ $1.99, p<.05)$. Sixty-eight percent of athletes who had sustained an injury over the preceding 12 months sustained at least one injury during the competitive season while $43.5 \%$ of athletes who did not have a recent history of injury sustained at least one injury during the competitive season.

\section{Hypothesis Testing}

Correlations. In contrast with Hypothesis 1, positive life stress and frequency of injury were negatively correlated $(r s=-.257, p<.05)$. There were no significant associations between positive life stress and severity of injury (time loss due to missed/modified practice) or between negative life stress and frequency or severity of injury. Contra to Hypothesis 2, emotion-focused coping was positively associated with severity of injury (time loss due to missed/modified 
practice $)(r s=.289, p<.05)$. Neither avoidance-coping nor the ACSI showed any significant associations with frequency or severity of injury. In contrast with Hypothesis 3, higher selfcompassion was significantly associated with higher negative life stress $(r s=.231, p<.05)$ and lower emotion-focused coping $(r s=-.259, p<.01)$ with small effect. In support of Hypothesis 3 , self-compassion was negatively associated with avoidance-focused coping $(r s=-.362, p<.01)$. Self-compassion showed no significant association with the ACSI coping measure. In accordance with Hypothesis 4, higher levels of self-compassion were moderately associated with lower levels of SAS Somatic Anxiety $(r s=-.436, p<.01)$ and SAS Worry $(r s=-.351, p<.01)$. As predicted by Hypothesis 5, lower scores on the negative LESCA were associated with higher levels of emotion-focused $(r s=-.242, p<.05)$ and avoidance-focused coping $(r s=-.249, p<$ .05). The ACSI Personal Resources Coping Score showed no significant association with either negative or positive life stress. A complete list of the bivariate analyses is displayed in Table 4 (See Appendix A).

Multiple Linear Regression Modeling. A multiple linear regression was calculated to predict frequency of injuries sustained during the competitive season based on self-compassion, life stress, coping and competitive sport anxiety. In contrast to Hypothesis 6, a non-significant regression equation was found $(F(7,73)=2.067, p=.057)$ with an $R^{2}$ of 0.167 . Positive LESCA (Beta $=-.292, p<0.05)$ was the only significant predictor while avoidance-focused coping $(\mathrm{Beta}=-.253, p=0.096)$ and emotion-focused coping $(\mathrm{Beta}=.218, p<0.101)$ were the next best predictors. A multiple linear regression was also calculated to predict time loss due to injuries sustained during the competitive season based on the same predictor variables. Time loss included any practices or competitions that were missed due to injury or where participation was modified due to injury. Contra to Hypothesis 6, a non-significant regression equation was found 
$(F(7,33)=1.069, p=.405)$ with an $R^{2}$ of 0.185. Emotion-focused coping $($ Beta $=.440, p<0.05)$ was the only significant predictor.

\section{Discussion}

\section{Study Overview}

The purpose of the current study was to investigate the contribution of self-compassion within the framework of antecedents to sport injury and the stress-injury association. Specifically, this study aimed to explore the role of self-compassion as a coping resource and a tool to cognitively reframe stressful sport and life experiences in collegiate athletes. It was hypothesized that this would in turn lead to reductions in frequency and severity of injury as is conceptually outlined by Williams and Andersen's (1998) stress-injury model. Over the course of the 2015 competitive season $48.7 \%$ of the athletes sustained at least one injury with $21.4 \%$ of athletes sustaining two or more injuries. Athletes who had sustained an injury over the preceding 12 months were more likely to incur an injury. Findings indicated that positive life stress was negatively associated with frequency of injury. Emotion-focused coping was positively associated with severity of injury while there were no associations between avoidance-focused coping or the ACSI and measures of injury. Other correlational analyses indicated that higher levels of emotion-focused and avoidance-focused coping were associated with lower levels of negative life stress. Results with respect to self-compassion indicated that higher levels of selfcompassion were significantly associated with higher negative life stress, and lower avoidancefocused and emotion-focused coping. SAS Worry and SAS Somatic Anxiety were negatively associated with self-compassion. Results from multiple linear regression modeling showed that self-compassion, life stress, coping and competitive sport anxiety were not significant predictors 
of frequency or severity of injury. Positive life stress was the sole significant predictor while avoidance-focused coping and emotion-focused coping were the next best predictors.

\section{Williams and Andersen's Stress-Injury Model}

This study provided further evidence in support of Williams and Andersen's (1998) multicomponent model of psychological stress and injury as well as introduced some evidence for the role of self-compassion in stress management for collegiate athletes. Firstly, the current study explored the contributions of all three of the areas that the Williams and Andersen model suggest are important in moderating the stress response i.e. history of stressors (history of injury and life stress), coping resources (coping style), and personality (competitive trait anxiety).

Over the course of the 2015 competitive season $48.7 \%$ of the athletes sustained at least one injury; $27.4 \%$ of athletes' sustained one injury, and $21.4 \%$ sustained two or more injuries. Injury rate was similar in football and volleyball at over $50 \%$ but was much lower in soccer at approximately $26 \%$. This is consistent with findings from a range of other studies that indicate that injury rates vary dependent on athletic population and type of sport, and that measurement of injury is also highly variable (Johnson \& Ivarsson, 2011; Johnson, 2007; Hanson et al., 1992). There was a significant difference in frequency of injury between athletes with a recent history of injury (12 preceding months) and those without a history of injury. Sixty-eight percent of athletes who had sustained an injury over the preceding 12 months sustained at least one injury during the competitive season while $43.5 \%$ of athletes who did not have a recent history of injury sustained at least one injury during the competitive season which confirms prior findings (Maddison \& Prapavessis, 2005; Smith, Smoll \& Ptacek, 1990a). Maddison and Prapavessis (2005) found that when athletes were high in avoidance-focused coping, had a history of injury, and were low in social support, then $31 \%$ of injury could be accounted for by negative life stress 
although these findings were non-significant. History of injury may impact susceptibility for reinjury for physical reasons such as incomplete recovery/healing of previous injury but also for psychological reasons such as high anxiety, worry and fear for re-injury (Williams, 2001) that may cause the attentional disruptions that contribute to injury susceptibility in Williams and Andersen's stress-injury model (1998). The findings from the current study further highlight the importance of considering history of injury in the stress-injury relationship.

The second component of Williams and Andersen's history of stressors category that this study explored was life event stress. Life event stress can refer to either positive or negative life event stress, each of which has their own total score within the LESCA. Findings indicated that, contrary to prior studies, positive life stress was negatively associated with frequency of injury and was a significant predictor of frequency of injury, with moderate effect size, in the multiple regression model (Johnson, 2007; Petrie, 1993; Hanson et al., 1992). There were no significant associations between negative life stress and frequency of injury. There has been some inconsistency in the literature in terms of the differential impact of negative and positive life stress on either frequency or severity of injury. That is, while some studies have pointed to the importance of negative life stress, others have emphasized the role of positive life stress in injury risk. With respect to positive life stress, findings from Hanson and colleagues (1992) exploration of Division I and II track and field athletes point to the positive association that may exist between positive life stress and frequency of injury. In Petrie's study of male collegiate Division I A football players $(N=158), 60 \%$ of injury variance could be explained by positive life stress, competitive anxiety and coping skills for athletes who were starters (Petrie, 1993). Although some other studies have found no contribution of positive life stress to injury variance, no prior studies have found a negative association between positive life stress and injury, which makes 
the findings from this study out of the ordinary. Specifically, according to Hanin's (2000) exploration of Individual Zones of Functioning (IZOF) and emotions that are either optimal or dysfunctional for injury outcome, certain emotions may promote complacency following a successful experience. This complacency will in turn reduce alertness and lower effort levels which may increase risk for injury (Johnson, 2007). However, the findings from this study imply that higher levels of positive life stress may be beneficial for reducing incidence of injury. That is, it suggests that the experience of positive life stressors may increase an athlete's overall wellbeing and mood as well as their self-confidence, which may in turn promote their attentional focus and engagement with their sport. This is consistent with Kleinert's (2007) findings that athletes higher in self-confidence had a lower odds ratio of injury incidence.

To better understand why positive life stress emerged as a positive predictor of lower incidence of injury, each individual item of the LESCA was looked at more closely. The top 20 items from the LESCA that had the highest frequency (i.e. the highest number of athletes indicated that they had experienced this life stressor over the preceding 12 months) were identified. Of the top 20 life stressors with the highest frequency, 13 were stressors that were experienced by the majority of athletes as a positive life stressor i.e. either 'somewhat positive', 'moderately positive', 'positive' or 'extremely positive'. Interestingly, there were several life stressors among these 13 life stressors that a large percentage of athletes indicated were 'extremely positive' or 'positive' that may have contributed to a lower incidence of athletic injury. Notably, many of these life stressors were directly associated with functioning/performance and well-being on the team. That is, at least greater than $60 \%$ of the athletes indicated that the following team-related life stressors were either 'extremely positive' or 'positive' events in their lives; 1) major change in relationship with coach (76\%) 2) major 
change in relationship with team mates (63.2\%) 3) major change in level of responsibility on team $(71.4 \%) 4)$ major change in level of athletic performance (69.2\%) 5) major change in playing status $(62.5 \%)$. This suggests that a positive experience with life stressors directly related to team cohesion, performance and the coach-athlete relationship may in fact serve as a buffer against susceptibility to injury.

Another life stressor of note that was experienced by the athletes as a primarily 'extremely positive' or 'positive' event (51.2\%) was the beginning of a new school experience (e.g. starting college or transferring). It is plausible that it would mostly be freshmen who would be beginning a new school experience and they might also be the athletes who get the least playing time so it therefore makes sense that they would have a lower incidence of injury. However, freshman did not have a significantly lower incidence of injury than the other year groups $[\mathrm{F}(4,112)=.501, \mathrm{p}=.735]$. Thus, beginning a new school experience may be a positive life event that reduces incidence of injury. Additionally, $98 \%$ of athletes who had received an athletic scholarship within the past 12 months indicated that it was either an 'extremely positive' or 'positive' event. Again, this was evenly distributed across freshmen, sophomores and juniors suggesting that it was not the freshman status or the amount of playing time that contributed to lower frequency of injury due to this positive life stressor. Andersen and Williams (1988) suggested that improvements in team cohesion can reduce susceptibility to injury because team cohesion is a way to manipulate social support, which has been found to have a buffering effect on risk for injury.

The finding that there were no significant associations between negative life stress and frequency of injury confirms results from some studies where negative life stress did not significantly predict frequency of injury (Ivarsson \& Johnson, 2010; Petrie, 1993; Hanson et al., 
1992) but it contrasts other studies that indicated that negative life stress significantly predicts incidence/frequency of injury (Laws-Gallien, 2001; Johnson \& Ivarsson, 2011). Specifically, Johnson and Ivarsson (2011) found that negative life stress accounted for $7 \%$ of variance in injury incidence in a sample of Swedish high school soccer players $(n=108)$. The LESCA is a difficult measure to administer because it is time-consuming to complete and it is often completed incorrectly. This could have played into the results found. Future studies may need to consider that although the LESCA is a very complete assessment of life and sport-related stress, there is a thin line between deciding whether or not it is worthwhile to use.

Although the current study used both frequency and severity (time loss) to assess injury, as suggested by Madisson and Prapavessis (2005), no association was found between either positive or negative life stress, and time loss due to injury. The majority of stress-injury studies have focused on frequency of injury and not severity of injury so the basis for comparison is small. However, Hanson and colleagues' (1992) found that as negative life stress increased, severity of injury increased, which would indicate that severity of injury and negative life stress are important to assess in athletes. Evidently, there is still a lot of room for further exploration of exactly what role positive and negative life stress play in frequency and time-loss due to injury.

According to Williams and Andersen's (1998) model, coping resources are also important to consider as they will moderate the stress-injury association when athletes successfully engage in coping strategies to reduce the physiological stress response. Correlational analyses from this study indicated that higher levels of emotion-focused and avoidance-focused coping were associated with lower levels of negative life stress which is consistent with theoretical stress and coping frameworks that outline the role of coping strategies in stress reduction ((Lazarus \& Folkman, 1984). However, it is interesting to evaluate the finding 
that avoidance-focused and emotion-focused strategies were negatively associated with lower negative life stress alongside the finding that participants who were higher in avoidance-focused coping also tended to be higher in emotion-focused coping with moderate/large effect. This would imply that athletes who displayed higher emotion-focused or avoidance-focused coping tended to generally have a higher capacity for coping i.e. they were high in coping all around. Subsequently, they may be able to change the coping strategy they employ dependent on the type of stressor they face.

This would be consistent with Lazarus and Folkman's (1984) transactional coping framework that outlines the situation-specific nature of coping strategies. That is, coping is dynamic, which means that the type of coping strategy employed could change over time and may also change due to type of challenge or stressor faced. Studies in elite athletes have indicated that coping strategies (approach vs. avoidant) will change dependent on the stressor (Anshel \& Gangyan, 2008) and also cross-culturally (Hoedaya \& Anshel, 2003). That is the selection of coping strategies (emotion-focused vs. problem-focused vs. "less useful strategies") will differ in athletes from different countries of origin as well as dependent on the type of stressor (Hoedaya \& Anshel, 2003). Emotion-focused coping strategies may therefore be more useful for dealing with situations where emotional distress needs to be managed (Stanton et al., 2000) while avoidant coping has been found to be useful when the stressful situation is uncontrollable (Roth \& Cohen, 1986). The LESCA does not give us any information about whether a stressor is controllable, changeable or can be internally attributed etc. It therefore follows that if the athlete would want to effectively manage any type of stressor they may face i.e. controllable vs. uncontrollable, they would need a wide range of coping strategies available to them and they would need to effectively change the coping strategy they employ dependent on 
the stressor. The positive association between emotion-focused and avoidance-focused coping as well as the negative association between these two forms of coping and lower level of negative life stress is conceptually plausible.

With regards to coping and positive life stress, positive life stress showed no significant association with either emotion or avoidance-focused coping or the ACSI. It is possible that when athletes experienced positive life stressors they did not engage in any coping strategies because they viewed the stressor as something good and therefore a situation that did not need any stress management. This may be supported by the previously mentioned finding that when positive life stress was higher, injury frequency was lower.

The role of coping in Williams and Andersen's stress-injury model is a two-part role. Firstly, effective coping helps the athlete adequately manage stress. Secondly, this will in turn reduce the physiological stress response that impacts risk of injury. Although previously discussed findings supported the role of coping in reducing stress, correlational findings between coping and injury are mixed and partly contradictory. That is, emotion-focused coping was positively associated with severity of injury, which means that when athletes engaged in higher levels of emotion-focused coping, they were at higher risk for greater time loss due to injury. This stands in contrast to evidence that outlines the protective role of coping resources in injury prediction (Wiese-Bjornstal, 2010; Johnson, 2007; Hanson et al., 1992; Smith, Smoll \& Ptacek, 1990). Additionally, there was no association between the ACSI and severity of injury which confirms some prior findings that there were no significant differences in coping resources (as measured by the ACSI-28) between injured and non-injured athletes (Johnson \& Ivarsson, 2011). Both of these findings question whether the evidence really is clear as to the role of coping in injury prevention. 
Results from multiple linear regression modeling indicated that although the predictive model was non-significant, which could have been due to small injury sample size, avoidancefocused coping was one of the strongest predictors of frequency of injury alongside positive life stress with moderate effect. That is, higher levels of avoidance focused coping were nonsignificantly predictive of lower incidence of injury. This is consistent with Williams and Andersen's conceptualization of the moderating impact of coping resources on the stress response and subsequent injury risk (Wiese-Bjornstal, 2010; Johnson, 2007; Hanson et al., 1992; Smith, Smoll \& Ptacek, 1990). However, findings from Maddison and Prapavessis (2005) study of injury in New Zealand rugby players found that life stress (negative), avoidance-focused coping, problem-focused coping and social support collectively accounted for $10-15 \%$ of injury variance. With respect to avoidance-focused coping, they theorized that this form of coping may in fact cause more distress if it is a situation/stressor that cannot be avoided e.g. competition yet the athlete is expending effort to try and avoid the situation. However, in the current study the direction of association was in the opposite direction, implying that engagement in avoidancefocused coping leads to lower risk for injury. Although this contradicts Maddison and Prapavessis (2005) findings, it is plausible that avoidance-focused strategies may be adaptive short-term solution for addressing a stressor that can be avoided. Roth and Cohen (1986) suggested that avoidance-focused coping strategies are useful as for short-term outcomes but may be more disruptive in the long-term. Nonetheless, Maddison and Prapavessis' reasoning may help explain the positive association between emotion-focused coping and severity of injury. That is, if athletes engaged in emotion-focused coping strategies in a situation where it may have been more appropriate to try and resolve the stressor itself, they may have caused themselves more distress in the long run. It is also plausible that the attempt itself to engage in a 
coping strategy to manage the stressor drew the athlete's attention away from their sport and towards the coping mechanism, which resulted in attentional disruption and increased susceptibility for injury. Roth and Cohen (1986) suggested that approach strategies and ventilation of affect, while useful for addressing controllable and changeable stressors, may also increase distress and nonproductive worry, which may help explain why emotion-focused coping positively predicted severity of injury.

Williams and Andersen's model also highlights the role of personality factors such as competitive trait anxiety. Results from this study indicated that SAS Worry and SAS Somatic Anxiety were positively associated with both emotion-focused and avoidance-focused coping. That is, participants higher in somatic anxiety and worry were also higher in both forms of coping. Similar to the discussion with stress, coping and injury, these results are seemingly counter-intuitive as higher levels of coping should result in lower levels of anxiety and worry. However, it is possible that somatic anxiety and worry were higher when ineffective coping strategies were engaged which may have caused more worry and anxiety instead of reducing stress. Furthermore, these findings are correlational so there is no way to determine whether higher coping resulted in higher anxiety or whether higher anxiety resulted in the engagement of more coping strategies. It would be similar for the opposite side of the correlation. That is, logically it makes sense that if anxiety was low, coping strategies may also be low because there is no need to engage in any coping strategies. This is a fundamental issue with correlational research. Interestingly, however, SAS Somatic Anxiety and SAS Worry were significantly lower in participants higher in ACSI Personal Resources Coping Score. This finding would be consistent with the theory that effective coping leads to reductions in anxiety and stress. With regard to multiple regression analyses, there were no significant findings in terms of the 
contribution of sport anxiety to injury susceptibility, which emphasizes the inconsistency of previous findings (Johnson, 2007; Petrie, 1993). On the one hand, Johnson and Ivarsson (2011) found that sport anxiety, as measured by the sport anxiety scale, significantly predicted $23 \%$ of variance in injury alongside life event stress, ineffective coping and mistrust. ANOVA analyses indicated that injured players had significantly higher levels of somatic trait anxiety than their non-injured counterparts. Ivarsson, Johnson and Podlog (2013) found that $24 \%$ of injury variance could be predicted by trait anxiety, negative life stress and daily hassles. The authors suggested that highly anxious individuals are more likely to experience an increased stress response due to a heightened likelihood for a threat appraisal when facing stressors. However, in Johnson's (2007) review of the stress-injury literature, it was found that research supporting the role of competitive trait anxiety in the stress-injury relationship is inconsistent and inconclusive. More research is therefore needed to clarify the moderating role of personality factors such as competitive trait anxiety in injury vulnerability.

\section{Stress, Coping, Injury and Self-Compassion}

Within the context of Williams and Andersen's (1998) stress-injury framework and the moderating effect of coping resources on injury susceptibility, self-compassion is being newly explored. The self-compassion literature consistently indicates that self-compassion is a useful coping tool to enhance adaptive coping, positive psychological functioning and well-being, and reduce anxiety in the face of stress (Neely et al., 2009; Neff, 2003a; Neff, Rude \& Kirkpatrick, 2007, Neff, 2003b; Neff, Kirkpatrick \& Rude, 2007; Allen \& Leary, 2010). However, the application of self-compassion in the sport context is a relatively new area of research. Several studies have looked specifically at the utility of self-compassion for women athletes in coping with negative events in sport (Mosewich et al., 2013), promoting positivity and responsibility 
(Ferguson et al., 2014), and reducing negative self-evaluative thoughts (Mosewich et al, 2011). However, the effect of self-compassion on measures of stress as well as different coping strategies had not yet been explored in athletes. Additionally, prior studies had not yet explored self-compassion in male athletes.

In contrast with the self-compassion literature outside of sport (Neely et al., 2009; Neff, 2003a; Neff, Rude \& Kirkpatrick, 2007, Neff, 2003b; Neff, Kirkpatrick \& Rude, 2007; Allen \& Leary, 2010), higher self-compassion was significantly associated with higher negative life stress. This finding is seemingly counter-intuitive as, from a conceptual standpoint, higher levels of self-compassion would promote a more mindful approach to health and performance that would allow athletes to adopt more balanced thoughts and expectations (Mosewich et al., 2013). However, the correlational nature of the findings means that they could also be interpreted as follows: when athletes experience higher levels of stress they are more likely to show themselves self-compassion. The experience of high levels of stress does not preclude someone from being a highly self-compassionate person. It may in fact be the case that an individual high in selfcompassion treats themselves much more kindly when their stress levels are high because they accept that sometimes they will feel stressed and there is no reason to feel shame or self-blame because of that stress. This effect or association may have been teased out if the study had included a scale to assess self-conscious emotions. For example, Mosewich and colleagues (2011) assessed self-conscious emotions using the Test of Self-Conscious Affect for Adolescents (TOSCA-A; Tangney, Wagner, Gavlas, \& Gramzow, 1991) which measures proneness to selfconscious emotions such as shame and guilt. They found that self-compassion was negatively associated with shame-proneness. 
Furthermore, if an athlete is more compassionate with themselves when they are stressed, as measured by the LESCA, this may be a mechanism through which subsequent anxiety, as measured by the SAS, about their stress can be reduced. That is, the mindfulness and acceptance component of self-compassion may allow the athlete to experience stress without also increasing anxiety levels. This would be consistent with the idea that self-compassion reduces anxiety in the face of stress (Neely et al., 2009; Neff, 2003a). In accordance with this hypothesis, higher levels of self-compassion were moderately associated with lower levels of SAS Somatic Anxiety and SAS Worry. The mindfulness subscale of the self-compassion scale also had the highest average score of the six subscales, which may further support the idea that acceptance and mindfulness allow a self-compassionate individual to successfully lower anxiety.

With reference to coping and self-compassion, previous research in the general population indicate that self-compassion is a form of emotional approach coping and is positively associated with the emotion-focused strategies of growth and acceptance and negatively associated with the avoidance-coping strategies of denial and mental disengagement (Neff, Hsieh \& Dejitterat, 2005). Within the context of coping, the findings from this study were two-fold. Firstly, higher levels of self-compassion were associated with lower emotion-focused coping with small effect, which is inconsistent with conceptualizations of self-compassion. The earliest research on self-compassion indicates that self-compassion, as a form of "emotional approach" coping, allows an individual to identify and express their emotions in a balanced and psychological adaptive way (Neff, 2003a). Additionally, the current study computed emotionfocused coping using the positive reframing and acceptance subscales of the Brief COPE, which have both been found to be significant correlates of self-compassion (Allen \& Leary, 2010; Neff, Hsieh \& Dejitterat, 2005). Specifically, positive reframing or cognitive restructuring is a central 
component of self-compassion that allows the individual to change their view of a stressful situation and view it with more optimism (Allen \& Leary, 2010). The second set of findings that self-compassion was negatively associated with avoidance-focused coping is consistent with prior research. Allen and Leary (2010) examined the role that self-compassion plays in the experience of negative life events and coping. Their review suggested that while avoidanceoriented coping strategies have been negatively related with psychological well-being and are therefore maladaptive, self-compassion is also negatively related with avoidance-coping strategies. By definition, self-compassionate individuals do not run away from their emotions and strive to be mindful and balanced in their experience of their emotions. That is, individuals high in self-compassion are more likely to accept responsibility for the mistakes yet treat themselves with kindness in the face of these failures. Perhaps even more importantly, selfcompassionate individuals tend to want the best for themselves which naturally leads to positive self-changes (Allen \& Leary, 2010). This may be especially relevant for athletes who inevitably face setbacks such as poor performance or defeat in a competition (Mosewich, Crocker \& Kowalski, 2013).

Interestingly, closer inspection of the subscales of the Brief COPE revealed that selfcompassion was significantly negatively associated with almost all 14 subscales which suggests that when self-compassion is high, all forms of coping are low. If self-compassion is its own, completely distinct form of coping then it may be plausible that when an individual employs self-compassion as their coping tool, there is no need to engage in any other coping strategies. However, this is contra to prior research that has found positive associations between selfcompassion and emotion-focused strategies such as acceptance and growth (Neff, Hsieh \& Dejitterat, 2005). This is also in contrast with the multi-component definition of self-compassion 
that includes some strategies that are assessed by the Brief COPE. However, the seemingly mixed results in this study with respect to self-compassion and coping are somewhat consistent with Allen and Leary's (2010) review of the research on self-compassion and different coping strategies. That is, with respect to approach and problem-focused strategies such as active coping, planning etc. (that are assessed by the COPE and Brief COPE) there have been some studies that found no significant association between self-compassion and problem-focused coping and other "approach" strategies. Allen and Leary suggested that while self-compassion is associated with active approaches to coping, self-compassionate individuals may only chose to engage in these strategies when they perceive the situation to be one that they can control and that can be resolved through action-taking. Furthermore, this study included 3 different measures of coping (i.e. Self-compassion, Brief COPE, and the ACSI). When one looks more closely at these three measures, they are all very distinct measures of coping that assess completely different constructs: a) self-compassion looks at self-kindness during times of perceived inadequacy and mindful awareness of emotions; b) the ACSI-28 measures psychological skills and not specifically “coping” skills (Crocker, Kowalski \& Graham, 1998); and c) the Brief COPE measures 3 different forms of coping: emotion-focused, problem-focused, and dysfunctional coping. Conceptually, each of these 3 measures is essentially looking at a different construct that may or may not be defined as 'coping', which have an effect on the differential relationship that each of them have on each other as well as on stress, coping and injury.

Furthermore, there is also the possibility that the culture surrounding sport may not allow for concepts such as self-compassion to be acknowledged and could have resulted in inaccurate or biased reporting of self-compassion levels, especially because the sample was predominantly male. Although self-compassion research indicates that the potential benefits of self-compassion 
make it a very promising avenue for athletes to pursue, some athletes have expressed concern that self-compassion may promote passivity and acceptance of mediocrity (Ferguson et al., 2014; Reis et al., 2015; Sutherland et al., 2014). Although the limited research on self-compassion in athletes has indicated that self-compassion may promote well-being through active engagement, taking initiative and responsibility (Ferguson et al., 2014), which is the opposite of passivity and mediocrity, the perception that self-compassion has no place in sport may still persist. Future studies may need to consider this and include an assessment of hyper-masculinity and sport ethic such as the Hypermasculine Values Questionnaire (HVQ; Archer, 2010). Although no gender differences were found in the levels of self-compassion, further studies may need to consider whether there is an interaction between gender and measures of conformity to sport ethic and attitudes of hyper-masculinity.

\section{Future Directions}

\section{Implications for Practice and Research}

This study primarily aimed to explore whether self-compassion could be a coping strategy for stress in collegiate athletes and subsequently potentially serve as a psychological protective factor for risk of athletic injury. Findings indicate that there may be some use for selfcompassion within the context of sport. Specifically, self-compassion may buffer the experience of anxiety in collegiate athletes and reduce the engagement of avoidance-focused coping strategies. However, several of the findings were in contrast with self-compassion research in the general population so there is certainly a need to further assess both levels of self-compassion in collegiate athletes as well as the association between self-compassion and measures of stress, anxiety and coping. 
This is the first study to date that has explored self-compassion in male athletes. The findings that there was no significant difference in level of self-compassion between the male and female athletes is noteworthy as this indicates that there may be a use for self-compassion with male athletes as well. More research is certainly needed to explore in more detail the role that self-compassion may play for male athletes both within the context of stress and coping as well as in injury susceptibility. Given that this study was the first known exploration of the application of self-compassion to injury prevention, more research in this area with both female and male athletes is needed as well as across different sports and competitive levels.

The prospective nature of this study leads to the conclusion that the next logical step for self-compassion research in athletes would be at the intervention level. Notably, self-compassion may be an appropriate psychological variable to study within the context of interventions for stress management and the stress-injury relationship because it is modifiable both through shortterm interventions such as self-compassion writing inductions (Leary et al., 2007) and longerterm interventions such as mindfulness-based stress reduction (MSBR; Chiesa \& Serretti, 2009), compassionate based therapy (CMT; Gilbert \& Proctor, 2006) and loving kindness meditation (LKM; Hofmann, Grossman \& Hinton, 2011). Although the aforementioned self-compassion intervention targeted stress and coping, and not injury, studies such as Maddison and Prapavessis' (2005) cognitive-behavioral stress management intervention study with athletes indicate that interventions that target stress can reduce susceptibility to injury. The authors targeted athletes who had an at-risk profile for injury and found that time loss due to injury and attentional disruption was reduced and coping skills were increased in the stress intervention group. The attentional changes were due to reduction in somatic anxiety and worry. This is noteworthy because the current study found that somatic anxiety and worry were negatively 
associated with self-compassion thereby indicating that self-compassion could be effective as an injury intervention tool through its impact on somatic anxiety and worry.

However, given the belief that seems to exist among athletes that self-compassion may promote passivity and acceptance of mediocrity (Ferguson et al., 2014; Reis et al., 2015), Reis et al. (2015) suggested that interventions and studies designed to promote self-compassion should take special consideration of the athlete's fear of self-compassion as this may lead them to resist the benefits of adopting a self-compassionate mindset. Rather, future studies that attempt to assess levels of self-compassion or induce self-compassion in athletes may want to provide athletes with a rationale for the benefits of self-compassion on well-being. Moreover, it may be worth presenting some information on the role that self-compassion plays in aiding an individual to learn from a failure experience rather than engage in ego defense mechanisms that obstruct learning. This may reduce reluctance on the athlete's part to reveal their own levels of selfcompassion. Furthermore, if the overall sporting culture cultivates low levels of self-compassion in athletes, an intervention to increase self-compassion may be particularly appropriate given Leary and colleagues' (2007) findings that self-compassion interventions are much more effective for individuals who are not already high in self-compassion. Low initial levels of selfcompassion would therefore not be an area of concern.

Additionally, self-compassion may be an appropriate construct to target for intervention given that it would be an inexpensive injury reduction technique that also has positive effects on overall well-being (Neely et al., 2009; Neff, 2003a; Neff, Rude \& Kirkpatrick, 2007, Neff, 2003b; Neff, Kirkpatrick \& Rude, 2007; Allen \& Leary, 2010), fear of failure and negative evaluation (Mosewich et al., 2011), eudemonic well-being (Ferguson et al., 2014) and negative cognitive states (Mosewich et al., 2013) in athletes. 


\section{Limitations}

This study is not without its limitations. At the outset, this study made several assumptions.

Firstly, it was assumed that Division II (DII) athletes would experience comparable stress levels to Division I athletes due to having to balance academic and social responsibilities with the demands of being an athlete. Secondly, there was an assumption that the measures chosen for this study would be appropriate to use with a sample of DII athletes. Additionally, the use of convenience sampling of DII athletes at a Mid-Atlantic university limits generalizability of findings to larger collegiate DII populations across the United States. As has been briefly mentioned previously, the correlational nature of this study does not allow for any conclusive statements to be made about the causal, directional or mediation effect that self-compassion may have on stress, anxiety, coping or injury. This should be considered when designing further explorations of self-compassion in collegiate athletes. Also worth noting is the fact that almost all of the pre-season data as well as the injury data were transformed for analysis given that there were violations of normality. Although transformations can be useful in reducing the risk for Type I or Type II errors in data sets that are not normal, data that has been transformed is difficult to interpret with respect to the original hypotheses. Additionally, the reliability of the self-compassion scale was lower than it has been found to be in prior research. That is, Cronbach's alpha was 0.705 as opposed to $\geq 0.86$ ) (Neff, 2003a; Neff et al., 2005). This may have to do with athletes' perceptions of self-compassion as an undesirable trait in sport. Moreover, all pre-season measures were self-report so there may have been some bias or a social desirability component with respect to reporting anxiety and stress levels as well as selfcompassion. The LESCA is also a difficult and long measure to complete and often has a very wide range of reported scores, as mentioned previously. Future studies may consider reducing 
the number of total measures administered when the LESCA is included in order to minimize the amount of time that athletes have to spend completing surveys that may contribute to improper completion of the LESCA. Additionally, it is worth noting that while sample size for pre-season measures was adequate, sample size for severity/time loss of injury $(n=41)$ was much smaller given that it was only the athletes who sustained an injury that could be included in these analyses. Power was subsequently very low. Having a much larger sample size to begin with may help increase the number of athletes that would be included in the severity of injury analyses. With respect to injury, there is a possibility that the injuries sustained by athletes were a re-occurrence of a previous injury, which was not checked in this study. This may introduce more of a physical reason for injury instead of a psychological reason. Williams (2001) outlined the importance of assessing for this, which future studies may want to explore.

Lastly, given that self-compassion, coping and all the other pre-season measured were only assessed at one time point, there was an underlying assumption in this study that scores on these measures would be consistent over time and would be predictive of subsequent stress and coping strategies later in the season. However, given Lazarus and Folkman's (1984) transactional approach to stress and coping, it is more likely the case that stress levels and coping strategies would have changed over time as well as dependent on the type of stressor that was faced. Furthermore, assessing coping at the beginning of a competitive season when stress levels may not be as high as in the middle of the season may result in inaccurate measurement of general coping strategies given that a low stress part of the season may not require high levels of coping. Williams and Andersen (1998) have also pointed to the need for assessing psychological states immediately prior to an injury. However, this is largely a very difficult feat to achieve as predicting when and where an injury will occur is limited. Future studies would need to 
determine how to disentangle the details surrounding the type of stressor faced and the subsequent coping strategy that is engaged as well as assess stress and coping at multiple timepoints across the season in order to get as close to a 'state' measure of coping and stress. A repeated measures design would be a stronger methodological design to capture state levels of coping and stress. Alternatively, a similar design could be followed as Kleinert's (2007) examination of mood states immediately prior to injury. Kleinert assessed psychological states such as self-confidence, positive mood, and calmness of athletes participating in sports at multiple different levels of competition. Measures were taken immediately prior to an aptitude test where participants were expected to reach certain targets of performance in various sport activities including swimming, track and field, and other team and individual sports. This design allowed for psychological states to be measured and then subsequent injury rates to be assessed within a 7 hour period of time (Kleinert, 2007).

\section{Conclusion}

This study aimed to provide an initial exploration of the application of self-compassion within the context of sport injury. It was theorized that within the context of Williams and Andersen's (1998) stress-injury model, self-compassion would predict more adaptive coping with stress and anxiety and may therefore reduce susceptibility to injury. In summary, this study provided some preliminary evidence for the role that self-compassion may play in the management of stress and anxiety in athletes. That is, self-compassion may lower the experience of somatic anxiety and worry within the collegiate context. This has important implications for minimizing the negative impacts of anxiety and stress within the context of athletic injury for both male and female athletes. Some evidence was also provided for the association between self-compassion and different forms of coping. Moreover, self-compassion was negatively 
associated with avoidance-focused coping, which may be beneficial in a stressful situation where action or acceptance is more adaptive than avoidance. However, there were several findings that were inconsistent with the self-compassion literature outside of sport. Additionally, there were no significant findings related to the role of self-compassion in injury risk reduction, which may be partly explained by a sporting culture that is resistant to concepts such as self-compassion. Nonetheless, given that this was a preliminary exploration of the application of self-compassion within the context of stress and injury, further investigation is certainly warranted. That is, there is justification to further investigate the applications of self-compassion in different athletic populations and sports as well as the implementation of self-compassion interventions.

This study also provided more evidence for the suitability of Williams and Anderson's (1998) stress-injury model as a model through which to examine the impact of stress, coping and personality factors on injury frequency and severity. Moreover, it is the combination of several predictors that is most useful in assessing risk for injury. However, this study highlighted some of the uncertainty and inconsistency that still exists surrounding the impact of different types of life stress i.e. positive or negative life stress, as well as the role of different types of coping strategies in managing stress and reducing the stress response involved in injury risk. Specifically, the finding that positive life stress may buffer susceptibility to injury suggests that the stress-injury association is highly complex. The differential impact of a set of predictors on frequency vs. severity of injury is also still unclear. Additional explorations of this theoretical framework are warranted to gain a clearer understanding of the precise role that stress, personality factors and coping play in injury susceptibility. 


\section{References}

Allen, A. B., Leary, M. R. (2010). Self-Compassion, Stress and Coping. Social and Personality Psychology Compass, 4/2, 107-118.

Andersen, M. B., \& Williams, J. M. (1988). A model of stress and athletic injury: Prediction and prevention. Journal of Sport and Exercise Psychology, 10(3), 294-306.

Andersen, M. B., \& Williams, J. M. (1999). Athletic injury, psychosocial factors and perceptual changes during stress. Journal of Sports Sciences, 17(9), 735-741.

Anshel, M. H., \& GangYan, S. (2008). Coping styles following acute stress in sport among elite Chinese athletes: a test of trait and transactional coping theories. Journal of Sport Behavior, 31(1), 3-21.

Archer, J. (2010). Derivation and assessment of a hypermasculine values questionnaire. British Journal of Social Psychology, 49(3), 525-551.

Blackwell, B., \& McCullagh, P. (1990). The relationship of athletic injury to life stress, competitive anxiety and coping resources. Athletic Training, 25, 23-27.

Brewer, B.W. (2001). Psychology of sport injury rehabilitation. In R. Singer, H. Hausen-blas \& C. Janelle's (Eds.), Handbook of research on sport psychology (2nd ed., pp. 787-804). Morgantown, WV: Fitness Information Technology

Burt, C. W., \& Overpeck, M. D. (2001). Emergency visits for sports-related injuries. Annals of emergency medicine, 37(3), 301-308.

Carver, C. S. (1997).You want to measure coping but your protocol's too long: Consider the Brief COPE. International Journal of Behavioral Medicine, 4, 92-100.

Carver, C. S., Scheier, M. F., \& Weintraub, J. K. (1989). Assessing coping strategies: a theoretically based approach. Journal of personality and social psychology, 56(2), 267. 
Chiesa, A. \& Serretti, A. (2009). Mindfulness-based stress reduction for stress management in healthy people: a review and meta-analysis. J Altern Complement Med, 15(5), 593-600.

Conn, J. M., Annest, J. L., \& Gilchrist, J. (2003). Sports and recreation related injury episodes in the US population, 1997-99. Injury prevention, 9(2), 117-123.

Cooper, C., Katona, C., \& Livingston, G. (2008). Validity and reliability of the brief COPE in carers of people with dementia: the LASER-AD Study. The Journal of nervous and mental disease, 196(11), 838-843.

Crocker, P. R., Kowalski, K. C., \& Graham, T. R. (1998). Measurement of coping strategies in sport. Advances in sport and exercise psychology measurement, 149-161.

Etzel, E. F. (2009). Counseling Student Athletes: Issues and interventions . Morgantown, WV: Fitness Information Inc

Edward, E.F., \& Watson, J.C. (2007). Ethical challenges for psychological consultations in intercollegiate athletics. Journal of Clinical Sport Psychology, 1, 304-317.

Etzel, E.F., Watson, J.C., Visek, A.J., \& Maniar, S.D. (2006). Understanding and promoting college student-athlete health: Essential issues for student affairs professionals. NASPA Journal, 43 (3), 518-546.

Ferguson, L. J., Kowalski, K. C., Mack, D. E., \& Sabiston, C. M. (2014). Exploring selfcompassion and eudaimonic well-being in young women athletes. Journal of sport \& exercise psychology, 36(2), 203-216.

Finch, C., \& Cassell, E. (2006). The public health impact of injury during sport and active recreation. Journal of Science and Medicine in Sport, 9(6), 490-497.

Folkman, S., \& Lazarus, R. S. (1980). An analysis of coping in a middle- aged community sample. Journal of Health and Social Behavior, 21, 219-239. 
Folkman, S., \& Lazarus, R. S. (1985). If it changes it must be a process: study of emotion and coping during three stages of a college examination. Journal of personality and social psychology, 48(1), 150.

Ford, I. W., Eklund, R. C., \& Gordon, S. (2000). An examination of psychosocial variables moderating the relationship between life stress and injury time-loss among athletes of a high standard. Journal of sports sciences, 18(5), 301-312.

Giacobbi, P., Jr., Foore, B., \& Weinberg, R. S. (2004). Broken clubs and expletives: The sources of stress and coping responses of skilled and moderately skilled golfers. Journal of Applied Sport Psychology, 16, 166-182.

Gilbert, P., \& Procter, S. (2006). Compassionate mind training for people with high shame and self-criticism: Overview and pilot study of a group therapy approach. Clinical Psychology \& Psychotherapy, 13, 353-379.

Gould, D. (1993). Intensive sport participation and the prepubescent athlete: Competitive stress and burnout. Intensive Participation in Children's Sports. Human Kinetics Publishers, Champaign, IL, 19-38.

Hanin, Y.L. (2000). Emotions in Sport. Champaign, IL: Human Kinetics

Hanson, S. J., McCullagh, P., \& Tonymon, P. (1992). The relationship of personality characteristics, life stress, and coping resources to athletic injury. Journal of Sport \& Exercise Psychology, 14, 262-272.

Hanton, S., Fletcher, D., \& Coughlan, G. (2005). Stress in elite sport performers: A comparative study of competitive and organizational stressors. Journal of Sports Sciences, 23(10), 1129-1141. 
Hanton, S., Wagstaff, C. R., \& Fletcher, D. (2012). Cognitive appraisals of stressors encountered in sport organizations. International Journal of Sport and Exercise Psychology, 10(4), 276-289.

Herman-Stabl, M. A., Stemmler, M., \& Petersen, A. C. (1995). Approach and avoidant coping: Implications for adolescent mental health. Journal of Youth and Adolescence, 24(6), 649665.

Hoedaya, D., \& Anshel, M. H. (2003). Use and effectiveness of coping with stress in sport among Australian and Indonesian athletes. Australian journal of psychology, 55(3), 159165.

Hofmann, S. G., Grossman, P., \& Hinton, D. E. (2011). Loving-kindness and compassion meditation: Potential for psychological interventions. Clinical psychology review, 31(7), 1126-1132.

Holt, N. L., \& Hogg, J. M. (2002). Perceptions of stress and coping during preparations for the 1999 women's soccerworld cup finals. The Sport Psychologist, 16, 251-271.

Hootman, J. M., Dick, R., \& Agel, J. (2007). Epidemiology of collegiate injuries for 15 sports: summary and recommendations for injury prevention initiatives. Journal of athletic training, 42(2), 311.

Ivarsson, A., \& Johnson, U. (2010). Psychological factors as predictors of injuries among senior soccer players. A prospective study. Journal of sports science \& medicine, 9(2), 347.

Ivarsson, A., Johnson, U., \& Podlog, L. (2013). Psychological predictors of injury occurrence: a prospective investigation of professional Swedish soccer players. Journal of sport rehabilitation, 22(1), 19-26. 
Johnson, U. (2007). Psychosocial antecedents of sport injury, prevention, and intervention: An overview of theoretical approaches and empirical findings. International journal of sport and exercise psychology, 5(4), 352-369.

Johnson, U., \& Ivarsson, A. (2011). Psychological predictors of sport injuries among junior soccer players. Scandinavian journal of medicine \& science in sports, 21(1), 129-136.

Junge, A. (2000). The influence of psychological factors on sports injuries review of the literature. The American Journal of Sports Medicine, 28(suppl 5), S-10.

Kaiseler, M., Polman, R., \& Nicholls, A. (2009). Mental toughness, stress, stress appraisal, coping and coping effectiveness in sport. Personality and individual differences, 47(7), 728-733.

Kleinert, J. (2007). Mood states and perceived physical states as short term predictors of sport injuries: two prospective studies. International Journal of Sport and Exercise Psychology, 5(4), 340-351.

Laws, G., \& Donna, Y. (2001). The relationship of life stress, social support systems, and coping behaviors associated with athletic injury and performance in women's intercollegiate softball teams. Dissertation Abstracts International, 62, 1133.

Lazarus, R.S. (1966). Psychological stress and the coping process. New York, NY, US: McGraw-Hill.

Lazarus, R.S.,\& Folkman, S. (1984). Stress, Appraisal, and Coping. New York: Springer.

Leary, M.R., Tate, E.B., Adams, C.E., Allen, A.B., \& Hancock, J. (2007). Self-compassion and reactions to unpleasant self-relevant events: The implications of treating oneself kindly. Personality and Individual Processes, 92, 887-904. 
Lysens, R. J., de Weerdt, W., Nieuwboer, A. (1991) Factors associated with injury proneness. Sports Med, 12, 281-289.

Maddison, R., \& Prapavessis, H. (2005). A psychological approach to prediction and prevention of athletic injury. Journal of Sport \& Exercise Psychology, 27, 289-310.

Mellalieu, S. D., Neil, R., Hanton, S., \& Fletcher, D. (2009). Competition stress in sport performers: Stressors experienced in the competition environment. Journal of Sports Sciences, 27(7), 729-744.

Mosewich, A. D., Crocker, P. R. E., Kowalski, K. C., \& DeLongis, A. (2013). Applying selfcompassion in sport: an intervention with women athletes. Journal of Sport \& Exercise Psychology, 35, 514-524.

Mosewich, A. D., Kowalski, K. C., Sabiston, C.M., Sedgwick, W.A., Tracy, J.L. (2011). SelfCompassion: A Potential Resource for Women Athletes. Journal of Sport \& Exercise Psychology, 33, 103-123.

Neely, M. E., Schallert, D. L., Mohammed, S. S., Roberts, R. M., \& Chen, Y. J. (2009). Selfkindness when facing stress: The role of self-compassion, goal regulation, and support in college students' well-being. Motivation and Emotion, 33(1), 88-97.

Neff, K. D. (2003a). The development and validation of a scale to measure self-compassion. Self and Identity, 2, 223-250.

Neff, K. D. (2003b). Self-Compassion: An Alternative Conceptualization of a Healthy Attitude Toward Oneself. Self And Identity, 2, 85-101.

Neff, K.D., Hsieh, Y.P., \& Dejitterat, K. (2005). Self-compassion, Achievement Goals, and Coping with Academic Failure. Self and Identity, 4, 263-287. 
Neff, K. D., Rude, S. S., \& Kirkpatrick, K. L. (2007). An examination of self-compassion in relation to positive psychological functioning and personality traits. Journal of Research in Personality, 41(4), 908-916.

Neff, K. D., Kirkpatrick, K. L., \& Rude, S. S. (2007). Self-compassion and adaptive psychological functioning. Journal of research in personality, 41(1), 139-154.

Neil, R., Hanton, S., Mellalieu, S. D., \& Fletcher, D. (2011). Competition stress and emotions in sport performers: The role of further appraisals. Psychology of sport and exercise, 12(4), 460-470.

Nippert, A. H., \& Smith, A. M. (2008). Psychologic stress related to injury and impact on sport performance. Physical medicine and rehabilitation clinics of North America, 19(2), 399418.

Nixon, H. L. (1993). Accepting the risks of pain and injury in sport: Mediated cultural influences on playing hurt. Sociology of Sport Journal, 10, 183-183.

Noblet, A. J., \& Gifford, S. M. (2002). The sources of stress experienced by professional Australian footballers. Journal of Applied Sport Psychology, 14(1), 1-13.

Passer, M. W. (1981). Children in sport: Participation motives and psychological stress. Quest, $33(2), 231-244$.

Petrie, T. A. (1992). Psychosocial antecedents of athletic injury: the effects of life stress and social support on female collegiate gymnasts. Journal of Behavioral Medicine, 18, 127138.

Petrie, T. A. (1993). Coping skills, competitive trait anxiety, and playing status: Moderating effects on the life stress injury relationship. Journal of Sport and Exercise Psychology, $15,261-274$. 
Pinkerton, R.S., Hinz, L.D., \& Barrow, J.C. (1989). The college student-athletre: Psychological considerations and interventions. Journal of American College Health, 37, 218-226.

Raes, F., Pommier, E., Neff, K. D., \& Van Gucht, D. (2011). Construction and factorial validation of a short form of the Self-Compassion Scale. Clinical Psychology \& Psychotherapy, 18, 250-255.

Reis, N. A., Kowalski, K. C., Ferguson, L. J., Sabiston, C. M., Sedgwick, W.A., Crocker, P.R. (2015). Self-compassion and women athletes' responses to emotionally difficult sport situations: An evaluation of a brief induction. Psychology of Sport and Exercise, 16 (3), $18-25$.

Roth, S., \& Cohen, L. J. (1986). Approach, avoidance, and coping with stress. American psychologist, 41(7), 813 .

Shamus, E. Kelleher, W. \& Foran, B. (2001). Basketball. In Shamus, E. \& Shamus, J. (Eds.), Sports Injury: Prevention \& Rehabilitation. (pp. 353-355). The McGraw-Hill Companies.

Smith, R. E., Schutz, R. W., Smoll, F. L., \& Ptacek, J. T. (1995). Development and validation of a multidimensional measure of sport specific psychological skills: the athletic coping skills inventory - 28. Journal of Sport and Exercise Psychology, 17, 379-398.

Smith, R. E., Smoll, F. L., \& Ptacek, J. T. (1990a). Conjunctive moderator variables in vulnerability and resiliency research: life stress, social support and coping skills, and adolescent sport injuries. Journal of personality and social psychology, 58(2), 360.

Smith, R.E., Smoll, F., \& Schutz, R. (1990b). Measurement and correlates of sport-specific cognitive and somatic anxiety: The Sport Anxiety Scale. Anxiety Research, 2, 263-380. 
Stanton, A. L., Kirk, S. B., Cameron, C. L., \& Danoff-Burg, S. (2000). Coping through emotional approach: Scale construction and validation. Journal of Personality and Social Psychology, 78, $1150-1169$.

Tangney, J.P., Wagner, P.E., Gavlas, J., \& Gramzow, R. (1991). The Test of Self-Conscious Affect for Adolescents (TOSCA-A). Fairfax, VA: George Mason University.

Uitenbroek, D. G. (1996). Sports, exercise, and other causes of injuries: results of a population survey. Research quarterly for exercise and sport, 67(4), 380-385.

Wiese-Bjornstal, D. (2010). Psychology and socioculture affect injury risk, response, and recovery in high-intensity athletes: A consensus statement. Scandanavian Journal of Medicine and Sciences in Sport, 20(Suppl. 2), 103-111.

Wiese-Bjornstal, D. M., Smith, A. M., Shaffer, S. M., \& Morrey, M. A. (1998). An integrated model of response to sport injury: Psychological and sociological dynamics. Journal of applied sport psychology, 10(1), 46-69.

Williams, J.M. (2001). Psychology of injury risk and prevention. In R.N. Singer, HA. Hausenblas, \& C.M. Janelle (Eds.), Handbook of sport psychology (2nd ed., pp. 766786). New York: Wiley \& Sons.

Williams, J. M., \& Andersen, M. B. (1998). Psychosocial antecedents of sport injury: Review and critique of the stress and injury model. Journal of applied sport psychology, 10(1), 525.

Yusoff, N., Low, W. Y., \& Yip, C. H. (2010). Reliability and validity of the Brief COPE Scale (English version) among women with breast cancer undergoing treatment of adjuvant chemotherapy: a Malaysian study. The Medical journal of Malaysia, (65), 41-4. 
Appendix A: Table 1. Sample Characteristics

\begin{tabular}{|c|c|c|c|}
\hline & Percent (n) & Mean (SD) & Range \\
\hline Age & & $19.50(1.57)$ & 18.00 to 26.00 \\
\hline \multicolumn{4}{|l|}{ Sport } \\
\hline American Football & $69.2 \%(81)$ & & \\
\hline Volleyball & $11.1 \%(13)$ & & \\
\hline Soccer & $19.7 \%(23)$ & & \\
\hline \multicolumn{4}{|l|}{ Education } \\
\hline High school diploma & $36.8 \%(43)$ & & \\
\hline Some college & $59.8 \%(70)$ & & \\
\hline College degree & $1.7 \%(2)$ & & \\
\hline Some graduate school & $0.9 \%(1)$ & & \\
\hline \multicolumn{4}{|l|}{ Race/Ethnicity } \\
\hline Black or African American & $40.2 \%(47)$ & & \\
\hline Asian or Asian American & $1.7 \%(2)$ & & \\
\hline White or Caucasian & $41.9 \%(49)$ & & \\
\hline Hispanic or Latino & $4.3 \%(5)$ & & \\
\hline American Indian/Alaskan Native & $1.7 \%(2)$ & & \\
\hline Other or Multiple Ethnicities & $10.3 \%(12)$ & & \\
\hline \multicolumn{4}{|l|}{ Gender } \\
\hline Male & $69.2 \%(81)$ & & \\
\hline Female & $30.8 \%(36)$ & & \\
\hline \multicolumn{4}{|l|}{ Year in School } \\
\hline Freshman & $35.9 \%(42)$ & & \\
\hline Sophomore & $25.6 \%(30)$ & & \\
\hline Junior & $24.8 \%(29)$ & & \\
\hline Senior & $11.1 \%(13)$ & & \\
\hline Graduate student & $2.6 \%(3)$ & & \\
\hline \multicolumn{4}{|l|}{ Playing Status on Team } \\
\hline Starter & $35.0 \%(41)$ & & \\
\hline Second String & $40.2 \%(47)$ & & \\
\hline Third String & $23.1 \%(27)$ & & \\
\hline \multicolumn{4}{|l|}{ History of Injury in Past Year } \\
\hline Yes & $21.4 \%(25)$ & & \\
\hline No & $78.6 \%(92)$ & & \\
\hline \multicolumn{4}{|l|}{ Severity of Previous Injuries } \\
\hline Minor (1-7 days) & $15.6 \%(5)$ & & \\
\hline Moderate (1 week to 1 month) & $28.1 \%(9)$ & & \\
\hline Major (>1 month) & $53.1 \%(17)$ & & \\
\hline \multicolumn{4}{|l|}{ Type of Previous Injuries } \\
\hline Ankle & $25.0 \%(8)$ & & \\
\hline Knee & $25.0 \%(8)$ & & \\
\hline Shoulder & $9.4 \%(3)$ & & \\
\hline Concussion & $15.6 \%(5)$ & & \\
\hline Back & $6.3 \%(2)$ & & \\
\hline Other & $6.3 \%(2)$ & & \\
\hline
\end{tabular}


Appendix A: Table 2. Descriptive Statistics

\begin{tabular}{|c|c|c|c|c|}
\hline & Mean (SD) & Range & $\mathrm{N}$ & $\alpha$ \\
\hline Athletic Coping Skills Inventory & & & & .863 \\
\hline Coping with Adversity & $6.95(2.26)$ & 2.00 to 12.00 & 112 & \\
\hline Coachability & $9.54(2.02)$ & 4.00 to 12.00 & 112 & \\
\hline Concentration & $7.19(2.17)$ & 2.00 to 12.00 & 112 & \\
\hline Confidence and Ach/ Motiv & $8.44(2.13)$ & 3.00 to 12.00 & 112 & \\
\hline Goal Setting and Mental Prep & $6.97(2.50)$ & 0.00 to 12.00 & 112 & \\
\hline Peaking under Pressure & $7.53(2.79)$ & 2.00 to 12.00 & 112 & \\
\hline Freedom from Worry & $6.61(2.62)$ & 0.00 to 12.00 & 112 & \\
\hline Personal Resources Coping & $53.35(10.54)$ & 32.00 to 78.00 & 112 & \\
\hline \multicolumn{5}{|l|}{ Score } \\
\hline \multicolumn{5}{|l|}{ Brief Cope } \\
\hline Self-distraction & $4.66(1.67)$ & 2.00 to 8.00 & 110 & \\
\hline Active Coping & $5.34(1.85)$ & 2.00 to 8.00 & 110 & \\
\hline Denial & $2.92(1.35)$ & 2.00 to 8.00 & 110 & \\
\hline Substance Use & $2.42(1.10)$ & 2.00 to 7.00 & 110 & \\
\hline Use of Emotional Support & $4.28(1.79)$ & 2.00 to 8.00 & 110 & \\
\hline Use of Instrumental Support & $3.95(1.80)$ & 2.00 to 8.00 & 110 & \\
\hline Behavioral Disengagement & $2.68(1.13)$ & 2.00 to 6.00 & 110 & \\
\hline Venting & $3.19(1.47)$ & 2.00 to 8.00 & 110 & \\
\hline Positive Reframing & $4.33(1.77)$ & 2.00 to 8.00 & 110 & \\
\hline Planning & $4.51(1.96)$ & 2.00 to 8.00 & 110 & \\
\hline Humor & $3.43(1.52)$ & 2.00 to 8.00 & 110 & \\
\hline Acceptance & $4.52(1.97)$ & 2.00 to 8.00 & 110 & \\
\hline Religion & $4.29(2.02)$ & 2.00 to 8.00 & 110 & \\
\hline Self-blame & $3.78(1.69)$ & 2.00 to 8.00 & 110 & \\
\hline Emotion-focused coping & $8.85(3.42)$ & 4.00 to 16.00 & 110 & .828 \\
\hline Avoidance-focused coping & $10.26(3.39)$ & 6.00 to 20.00 & 110 & .756 \\
\hline \multicolumn{5}{|l|}{ Sport Anxiety Scale (SAS) } \\
\hline Somatic Anxiety & $16.13(5.61)$ & 9.00 to 34.00 & 117 & .858 \\
\hline Worry & $14.25(5.11)$ & 7.00 to 28.00 & 117 & .867 \\
\hline Concentration Disruption & $7.77(2.61)$ & 5.00 to 18.00 & 117 & \\
\hline Self-compassion Score & $18.84(3.56)$ & 6.00 to 28.50 & 113 & .705 \\
\hline Self-kindness Score & $2.87(0.96)$ & 1.00 to 5.00 & 113 & \\
\hline Self-judgment Score & $2.52(1.13)$ & 1.00 to 5.00 & 113 & \\
\hline Common Humanity Score & $2.60(0.92)$ & 1.00 to 5.00 & 113 & \\
\hline Isolation Score & $2.67(1.14)$ & 1.00 to 5.00 & 113 & \\
\hline Mindfulness Score & $3.34(0.99)$ & 1.00 to 5.00 & 113 & \\
\hline Over-identification Score & $2.78(1.00)$ & 1.00 to 5.00 & 113 & \\
\hline \multicolumn{5}{|l|}{ Life Events Survey for Collegiate } \\
\hline \multicolumn{5}{|l|}{ Athletes (LESCA) } \\
\hline LESCA Total Score & $32.63(27.00)$ & 3.00 to 170.00 & 87 & \\
\hline LESCA Negative Score & $-15.17(18.06)$ & -122.00 to 0.00 & 87 & \\
\hline LESCA Positive Score & $17.46(15.87)$ & 0.00 to 74.00 & 87 & \\
\hline
\end{tabular}


Appendix A: Table 3. Descriptive Injury data from competitive season

\begin{tabular}{|c|c|c|c|}
\hline & Percent (n) & Mean (SD) & Range \\
\hline \multicolumn{4}{|l|}{ Frequency of Injury } \\
\hline None & $51.3 \%(60)$ & & \\
\hline One & $27.4 \%(32)$ & & \\
\hline Two & $15.4 \%(18)$ & & \\
\hline Three & $5.1 \%(6)$ & & \\
\hline Four & $0.0 \%(0)$ & & \\
\hline Five & $0.9 \%(1)$ & & \\
\hline \multicolumn{4}{|l|}{ Type of Injury } \\
\hline Ankle/Foot & $16.5 \%(15)$ & & \\
\hline Knee & $12.1 \%(11)$ & & \\
\hline Shoulder & $20.8 \%(19)$ & & \\
\hline Concussion & $5.5 \%(5)$ & & \\
\hline Back & $3.3 \%(3)$ & & \\
\hline Other & $41.8 \%(38)$ & & \\
\hline Time Loss due to Injury (Missed/Mod) & & $9.84(13.28)$ & $0.00-58.00$ \\
\hline $\begin{array}{l}\text { Time Loss due to Injury (Go as Able } \\
\text { Included) }\end{array}$ & & $18.25(15.90)$ & $0.00-64.00$ \\
\hline
\end{tabular}


Appendix A: Table 4. Spearman's Rho examining associations between pre-season study variables

\begin{tabular}{|c|c|c|c|c|c|c|c|c|c|c|c|}
\hline & 1. & 2. & 3. & 4. & 5. & 6. & 7. & 8. & 9. & 10. & 11. \\
\hline 1. LESCA Negative Stress & 1.00 & & & & & & & & & & \\
\hline 3. Emotion-focused Coping & $-.242 *$ & .120 & 1.00 & & & & & & & & \\
\hline 5. Self-Compassion Score & $.231 *$ & -.069 & $-.259 * *$ & $-.362 * *$ & 1.00 & & & & & & \\
\hline 6. SAS Worry & $-.337 * *$ & .068 & $0.350 * *$ & $.450 * *$ & $-.351 * *$ & 1.00 & & & & & \\
\hline 7. SAS Somatic Anxiety & -.069 & -.036 & $0.228 *$ & $.415^{* *}$ & $-.436 * *$ & $.601 * *$ & 1.00 & & & & \\
\hline 10. Severity of Injury 1 & -.083 & -.037 & $.289^{*}$ & .118 & -.061 & .113 & .191 & .108 & $-.292 *$ & 1.00 & \\
\hline 11. Severity of Injury 2 & -.082 & -.025 & .136 & .117 & .040 & -.003 & -.025 & .174 & $-.534 * *$ & $.661 * *$ & 1.00 \\
\hline
\end{tabular}

Note: Severity of Injury 1 refers to total time loss due to injury, which includes practices or competitions missed or modified. Severity of Injury 2 refers to total time loss due to injury, which includes practices or competitions missed or modified as well as days where the athlete could self-determine their involvement in practice/competition.

$* \mathrm{p}<.05 * * \mathrm{p}<.01$ 


\section{Appendix B: Extended Literature Review}

\section{Introduction}

The positive psychology movement has shifted the focus of assessments of well-being from the absence of psychopathologies to the presence of psychological variables that promote resilience and strength and emphasize human potential rather than weaknesses (Seligman \& Csikszentmihalyi, 2000). That is, humans have an immeasurable capacity for hope and optimism, and display a range of positive individual traits such as perseverance, forgiveness, spirituality and courage that can serve as very powerful protective factors against pathologies such as anxiety, depression and stress (Boscaglia, Clarke, Jobling, \& Quinn, 2005; Seligman \& Csikszentmihalyi, 2000). This literature review will focus specifically on self-compassion as a positive psychological variable with important potential applications within the athletic context. Self-compassion is conceptualized as a form of self-acceptance that is warm and healthy (Neff, 2003a). It is composed of three distinct parts: 1) kindness and understanding to oneself in times of suffering and failure; 2) common humanity as opposed to isolation, i.e. suffering and perceived inadequacy are parts of the shared human experience; and 3) balanced awareness of one's emotions instead of over-identification (Neff, 2003a; Neff, 2003b). Self-compassion draws upon Eastern Buddhist philosophies of a healthy self-attitude and is based in the concept of mindfulness (Neff, 2003b). Mindfulness contributes to all three components of self-compassion by facilitating acceptance and balance of emotions, promoting interconnectedness and a nonjudgmental view of the self (Neff, 2003a). Self-compassion is associated with increased levels of well-being and a number of positive outcomes such as lower levels of anxiety and depression that will be further discussed in this literature review (Neely et al., 2009; Neff, 2003a). Studies of self-compassion in relation to positive psychological functioning have demonstrated the 
association between self-compassion and optimism, positive mood, curiosity, and exploration (Neff et al., 2007a).

This literature review will explore the applicability of self-compassion within the collegiate athletic context. Specifically, the goal of this review is to highlight the relevance of self-compassion as a potential resource for collegiate athletes in coping with psychological stress and subsequently its possible contribution to incidence of athletic injury. Firstly, psychological stress will be defined and discussed within the framework of coping strategies. This discussion will include an integration of current knowledge on coping strategies employed by athletes and the significance of appraisal of stress in exacerbating the experience of stress. Secondly, the outcomes of psychological stress and maladaptive coping strategies will be explored in relation to athletic injury. This will be followed by a brief description of the importance of psychological predictors of injury as well as an exploration of Andersen and Williams (1988) stress-injury model. Next, the literature on self-compassion will be briefly reviewed to provide a foundation for the discussion of self-compassion in relation to psychological stress, appraisal and coping in athletes. This will include a review of current applications of self-compassion within the athletic context. Following the separate discussions of psychological stress, coping, injury, and selfcompassion, the associations between these four areas within the sporting context will be addressed. That is, an argument will be presented for the potential role of self-compassion as a stress buffer and as an adaptive coping strategy for collegiate athletes. This will be followed by a discussion of the subsequent positive impact that stress reduction through the use selfcompassion may have on incidence of athletic injury.

\section{STRESS AND COPING}

\section{Link Between Stress and Coping Strategies}


There is extensive literature detailing the association that exists between stress, coping strategies and psychological adjustment (Carver et al., 1989; Lazarus, 1966; Lazarus \& Folkman, 1984). Lazarus (1966) developed a theory of psychological stress and coping that involved three processes of stress. The first two processes are concerned with cognitive appraisal (i.e. how the individual evaluates the threat and their ability to respond to the threat): (a) primary appraisal where the individual first perceives a threat to themselves and their well-being; and (b) secondary appraisal where the individual decides whether they are able to and how they will respond to the threat. The third process is coping and is distinct from appraisal in that it is the stage where an individual executes their response to the threat. Coping is defined as the thoughts or actions employed by an individual to manage a situation that is perceived to be stressful, which is largely affected by the individual's perceptions of their capacity to respond to the stress (Carver et al., 1989; Lazarus, 1966; Lazarus \& Folkman, 1984).

Coping is addressed from two theoretical frameworks, trait and transactional theory (Coats, Hoppmann \& Scott, 2014). Trait theory dictates that individuals will respond in a consistent manner to stressors across situations (Carver, Scheier \& Weintraub, 1989). That is, emphasis is placed on the dispositional nature of coping. Transactional theory states that coping preferences are a result of interactions between person and situational factors (Lazarus \& Folkman, 1984; Parkes, 1986). This literature review will focus on transactional theory given the weak associations that have been found between situation and coping style in studies using trait theory (Coats, Hoppmann \& Scott, 2014).

Transactional theory focuses on the importance of the individual's appraisal of the situational factors and how these factors may impact their well-being (Lazarus \& Folkman, 1984). That is, a situation itself cannot be stressful; the interaction between "person" and 
"situation" factors will result in a stress appraisal. In this way, coping is a dynamic, situationspecific process (Folkman et al., 1986). According to Lazarus and Folkman, the stressor must have personal significance or meaning to the individual in order for it to be perceived as stressful (Lazarus \& Folkman, 1984; Park \& Folkman, 1997). The meaning (person factors) of the situation is influenced by goals at stake, beliefs of the individual's place in the environment, and personal resources (Lazarus, 1999). Furthermore, meaningful situations will be appraised as stressful if they also include one of the following eight properties: 1) imminence: length of anticipation prior to an event, 2) predictability: whether expectancies of an event are met, 3) novelty: prior similar experience with situation, 4) duration: length of the event, 5) event uncertainty: likelihood of the occurrence of an event, 6) temporal uncertainty: timing of event is not known for certain, 7) timing in relation to life cycle: simultaneous occurrence of other stressful events, and 8) ambiguity: situation is unclear so information needed for appraisal is unclear. Thus, several factors contribute to an individual's appraisal of a situation as stressful, which include situation and person factors (Lazarus \& Folkman, 1984).

The coping literature also makes distinctions between different forms of coping. Lazarus and Folkman (1984) differentiated between problem-focused coping and emotion-focused coping (Carver et al., 1989; Folkman \& Lazarus, 1980). Problem-focused coping involves trying to alleviate the source of stress while emotion-focused coping involves managing the emotional distress associated with the situation. There is empirical support that both forms of coping are used when responding to a stressful situation (Folkman \& Lazarus, 1980; Folkman \& Lazarus, 1985). Carver, Scheier, and Weintraub (1989) studied coping strategies in undergraduate students $(n=156)$ at the University of Miami. Participants were asked to recall the most stressful event that had occurred over the preceding 12 months. They then completed the situationally 
framed items of the COPE (Carver, Scheier \& Weintraub, 1989) in order to assess which coping strategies they relied on to deal with the stressful event. Findings indicated that problem-focused coping strategies such as planning, active coping, and suppression of competing activities occurred more often in controllable situations (i.e. situations amenable to change) whereas emotion-focused coping strategies such as acceptance occurred more in uncontrollable situations. Furthermore, if a stressful event was rated as more important, the individual was more likely to engage in emotion-focused strategies such as venting of emotions and seeking of emotional support (Carver et al., 1989). These results are consistent with findings from Folkman and Folkman and Lazarus' (1980) exploration of problem and emotion-focused coping strategies in response to stressful daily events in a sample of older adults $(n=100)$. Folkman and Lazarus (1985) further replicated these findings in their investigation of coping in undergraduate students ( $n=261)$ who were experiencing mid-term examination stress. Additionally, while Carver and colleagues (1989) used the COPE as their primary measure of assessment, the latter two studies used the 68-item Ways of Coping checklist (WCC; Folkman \& Lazarus, 1980) and a specially designed Stress Questionnaire respectively (Folkman \& Lazarus, 1980; Folkman \& Lazarus, 1985). The similarity in findings across different measures is strong evidence for the reliability of the results with regard to variations in adoption of problem vs. emotion-focused coping strategies. Although some studies have found that emotion-focused coping may be the more maladaptive form of coping (Saklofske et al., 2012), it is also argued that emotion-focused coping may promote psychological adjustment through awareness and understanding of emotions (Stanton et al., 2000). Focusing on emotions may in fact allow the individual to reappraise the stressor and come to a healthier attribution of the event (Stanton et al., 2000). 
Problem vs. emotion-focused coping strategies can be assessed using the COPE which measures 13 conceptually distinct styles of coping (Carver et al., 1989). The 13 COPE scales are divided into 5 problem-focused strategies (active coping, planning, suppression of competing events, restraint coping, seeking social support for instrumental reasons), 5 emotion-focused strategies (seeking social support for emotional reasons, positive reinterpretation and growth, acceptance, turning to religion, denial) and 3 less useful coping strategies (behavioral engagement, mental disengagement, and focus on or venting of emotions) (Carver et al., 1989). A shorter version of the COPE has since been developed and is one of the most frequently used coping measures (Kasi et al., 2012). The Brief COPE inventory (Brief COPE; Carver, 1997) is a 28-item survey that measures three conceptually distinct forms of coping: (a) problem-focused coping, (b) emotion-focused coping and (c) dysfunctional coping. There are fourteen subscales (active coping, planning, positive reframing, acceptance, humor, turning to religion, using emotional support, using instrumental support, self-distraction, denial, venting, substance use, behavioral disengagement, and self-blame) with two items on each subscale. Each item is scored on a four-point Likert scale ( $1=\mathrm{I}$ haven't been doing this at all to $4=\mathrm{I}$ have been doing this a lot). Reliability and validity statistics have consistently been found to be adequate in various non-sport populations; Cronbach's alpha ranges from 0.5 to 0.9 (Carver, 1997; Cooper, Katona \& Livingston, 2008; Yusoff, Low \& Yip, 2010). Carver suggests that subscales can be selected individually according to preference for data analysis (Carver, 1997).

The coping literature also differentiates between active (approach) and avoidant coping strategies whereby active coping involves trying to change the stressor itself (behavioral coping) or changing the way you think about the stressor (psychological) and avoidant coping involves psychologically or behaviorally avoiding the stressful situation through mental states such as 
withdrawal or activities such as alcohol (Carver et al., 1989; Herman-Stabl, 1995). Generally, active/approach coping is associated with better emotional adjustment to stressful events and a lower experience of depression than avoidant coping strategies (Carver et al., 1989; HermanStabl, 1995).

However, the effectiveness of approach vs. avoidant coping is not as clear-cut as these studies may suggest and instead reflects a transactional approach to coping. Approach and avoidant coping have been found to be more or less appropriate in certain situations (Roth \& Cohen, 1986). Roth and Cohen (1986) suggested that evaluations of coping effectiveness depend on three factors: 1) the controllability of the stressful situation, 2) the fit between the coping style and the situational demands, and 3) the timing of the evaluation of the coping style. From their review of the coping literature, Roth and Cohen (1986) found support for the use of avoidant coping when the situation is uncontrollable, and approach coping when the situation is controllable and the individual can act to change the situation. Furthermore, if the individual's coping style matched the situational demands, less distress was experienced. Lastly, avoidance strategies seem to be more effective for short-term outcomes while approach strategies were better for long-term outcome measures. Therefore, both approach and avoidance coping have costs and benefits. While approach strategies may allow for appropriate action to be taken and for ventilation of affect, they may also increase distress and nonproductive worry. However, while avoidance strategies may temporarily reduce stress and increase courage and hope, they may also increase disruptive avoidance behaviors, increase emotional numbness, and promote a lack of awareness of the relationship between symptoms and trauma. They concluded that demonstrations of flexibility regarding approach and avoidant coping would be most adaptive (Roth \& Cohen, 1986). In a meta-analysis of 43 studies using avoidant and approach (non- 
avoidant) strategies, Suls and Fletcher (1985) found similar results indicating that avoidant strategies are more effectives on a short term basis and approach strategies are more effectives for long-term outcomes. This same pattern has also been found in physical adaptations to approach vs. avoidant coping strategies (Mullen \& Suls, 1982).

\section{STRESS AND COPING, AND SPORT}

\section{Stress and Sport}

Psychological stress is an intrinsic component of playing competitive sport regardless of the level of competition (Hanton, Fletcher \& Coughlan, 2005; Mellalieu et al, 2009; Noblet \& Gifford, 2002; Gould, 1993; Passer, 1981) or the sport (Giacobbi, Foore \& Weinberg, 2004; Gould, Jackson \& Finch, 1993; Holt \& Hogg, 2002). However, high levels of stress in athletes has been linked to breakdowns in performance where well-learned skills can no longer be executed i.e. choking (Baumeister, 1984; Hardy, 1999; Smith et al., 2000). Several different models of stress have been used within the sport psychology literature (Gill, 1994). Drive theory (Spence, 1971) and the inverted-U hypothesis (Martens \& Landers, 1970) were two of the more dominant theories that described the link between arousal levels and performance (Gill, 1994). More cognitive approaches to sport-related stress were later developed from Spielger's (1966; 1989) work that focused on competitive anxiety. Sport-specific measures of trait (Martens, 1976) and state anxiety (Martens et al., 1990) were developed to investigate the unique impact of anxiety on sport performance (Gill, 1994).

Several specific measures have also been developed to assess life event stress experienced by athletes. The Daily Analysis of Life Demands for Athletes (DALDA: Rushall, 1990) and the Perceived Stress Scale-10 (PSS-10; Cohen, Kamarck, \& Mermelstein, 1983) are 9 and 10-item scales respectively that assess stressors that an athlete may face. However, both of 
these scales do not assess sport-specific stressors (Lu et al., 2012). The Life Event Scale for Collegiate Athletes was developed by Petrie (LESCA; 1992) to assess which life event stressors (including sport-specific stressors) an athlete has experienced during the previous twelve months. The LESCA is a 69 item survey that assesses an athlete's response to an event on an eight-point Likert scale $(-4=$ extremely negative to $+4=$ extremely positive). An example item includes "major change in playing status on a team". Scores on each item are summed to give three different life event scores: negative (scores range from 0 to -276), positive (scores range from 0 to +276 ) and total (sum of positive and negative scores). This instrument was developed using intercollegiate athletes and has been shown to have excellent psychometric properties including moderate criterion-related validity between positive and negative life stress scores $(\mathrm{r}=.55$, $\mathrm{p}<0.05)$ (Petrie, 1993), high test-retest reliability (.76-.84) (Petrie, 1993), and significant construct validity (Petrie, 1992; Petrie, 1993; Andersen \& Williams, 1999). The LESCA is commonly used to assess history of stressors in athletes (Johnson \& Ivarsson, 2011; Steffen, Pensgaard \& Bahr, 2009).

Collegiate athletes are particularly susceptible to elevated levels of psychological stress due to the unique demands they face in trying to balance the academic, psychosocial and athletic domains of their college experience (Etzel, 2009; Lu et al., 2012). Not only do they experience similar challenges to non-collegiate athletes such as interpersonal conflict, adjustment to college, and substance abuse issues they also face stressors related to their sport that have the potential to impact their mental well-being, their immune functioning and their sport performance (Etzel, 2009; O’Leary, 1990; Yusko et al., 2008). There are multiple different sources of stressors in competitive sport that have the potential to impact an athlete's well-being and performance. In a study of Division I athletes $(n=14)$ at a Mid-Western University, Kaiseler, Polman and Nicholls 
(2009) found that athletes' experiences of stress result from participation in collegiate sport; athletes revealed that although athletic participation was enjoyable and was often a way to cope with daily stress, sport itself was also a source of stress. Specifically, athletes cited sport as being stressful for two reasons. The first was when they were in sporting situations where they had no control over the people, the situation or their own experience. This included situations that involved their teammates or coaches, a lack of sense of competence, gender stereotypes and social inequality. The second was when there was a lack of social support for non-athletic endeavors or they felt excluded from the rest of the student body. Findings from this study emphasize the importance of promoting coping strategies in athletes and making coping resources easily accessible to them (Kaiseler, Polman \& Nicholls, 2009).

Sources of stress for athletes have been categorized in terms of competition/performance stress and organizational stress (Hanton, Fletcher \& Coughlan, 2005; Mellalieu et al, 2009; Noblet \& Gifford, 2002). Several studies have noted that both elite (have represented their country on an international level) and non-elite athletes (competing at the national, district or university level) face an array of similar (although some different) stressors prior to competing such as technical and physical preparation, risk of injury, internal and external expectations, selfpresentation to coaches, team-mates and spectators, and rivalry (Mellalieu et al, 2009). Similarly, they face common organizational stressors such as factors intrinsic to the sport (e.g. competition format, weather and facilities), roles in the sport organization (i.e. responsibility), sport relationships and interpersonal demands (e.g. leadership, personality type, interactions etc.), athletic career and performance development issues, organizational structure and climate of the sport (Hanton, Fletcher \& Coughlan, 2005; Mellalieu et al, 2009; Noblet \& Gifford, 2002). However, elite performers (competing at the international level for at least 5 years) have been 
found to face more organizational stressors than non-elite sport performers (competing at the district or regional level) (Fletcher et al., 2012). Although collegiate athletes are technically not considered elite athletes within the definition of international travel and competition, they certainly face many similar stressors to elite athletes such as risk for injury or playing while injured, training and competition environment, national travel, pressure to perform, media scrutiny etc. due to the presence of stressors that are inherent to sport irrespective of level of competition (Mellalieu et al., 2009).

More recently, researchers have incorporated the key features from Lazarus' psychological stress model thereby emphasizing the cognitive appraisal aspect of stress, the complexity of stress, as well as the focus that should be placed on individual characteristics when dealing stress (Gill, 1994). Thatcher and Day (2008) examined whether Lazarus and Folkman's (1984) eight underlying properties of a stressor influence whether a meaningful situation is appraised to be stressful within a sporting context. Eight female and eight male national trampolinists were recruited and participated in semi-structured qualitative interviews. Results indicated that all eight underlying properties were applicable to stress experienced by athletes. Furthermore, self and other comparison and inadequate preparation were found to be additional underlying properties that caused stress. The authors concluded that stress could be avoided in relation to all eight properties if: 1) novelty: the athlete re-frames their appraisal of the novelty of the competitive situation, then they would be able to draw on other experiences to deal with the stressor, 2) predictability: athletes prepare for unexpected change in order to reduce stress, 3) event uncertainty: athletes are aided in finding objective probabilities for events rather than subjective probabilities from personal experience, 4) imminence: athletes use pre-competition routines and confidence to reduce stress, 5) duration: athletes are taught how to use positive self- 
talk to eliminate stress, 6) temporal uncertainty: athletes use relaxation techniques, 7) ambiguity: the athlete is encouraged to seek out as much information about the event as possible beforehand, and 8) timing in relation to life cycle: athletes use time management and social support to deal with general stress. Thatcher and Day's study suggests that stressful appraisals within the athletic context may be reduced if the underlying properties of stress are addressed.

Studies on stress in elite athletes have also directly examined the role of cognitive appraisal of organizational stressors. Hanton, Wagstaff, and Fletcher (2012) examined daily diary entries of stressors encountered by four elite sport performers across a period of six weeks. Response to stressors were divided into reflections on the nature of the stressor i.e. cause and whether it was unexpected and controllable, appraisal of the stressor which included evaluations and thoughts, and responses to the stressor. Findings indicated that athletes predominantly appraised the organizational stressors as out of their control and threatening or harmful, rather than challenging. Additionally, $42 \%$ of the stressors that they appraised to be threatening were organizational stressors that are intrinsic to the sport e.g. training load and environment. The athletes further noted that they perceived a limited availability of coping resources to deal with the threatening stressors. Moreover, when stressors were re-appraised as being challenging rather than threatening, athletes tended to "take action" against the stressor or "get on with it". Hanton and colleagues discussed the importance that individual differences such as resilience and mental toughness may play in the re-appraisal process.

Additionally, Neil et al (2011) examined appraisals and emotional reactions in six elite and six non-elite athletes across several different sports. Findings indicated that an athlete's emotional response to a stressor can also be re-appraised and used in a facilitative manner e.g. anxiety can be beneficial for performance if the athlete re-appraises the anxiety as a sign that 
they want to do well, and therefore increase their concentration and competition efforts.

Conversely, a negative re-appraisal of an emotion can lead to further debilitating effects (Neil et al., 2011). These findings from Hanton et al. (2012) and Neil et al. (2011) are significant because they highlight the importance of the process of cognitive appraisal of stressors and emotional responses to stressors and imply that interventions or psychological variables that intervene at the appraisal stage of the stress response could moderate the impact that stressors have on elite athletes and possibly collegiate athletes. Thus, in situations where the stressor cannot be changed (e.g. stressors intrinsic to sport such as training load) the stress response can be reduced by focusing on minimizing threat appraisals of stressors and instead facilitating challenge appraisals. This could have significant implications on injury risk reduction strategies. Therefore, further exploration of stress reduction techniques that target appraisal of stress collegiate athletes is warranted.

\section{Coping Strategies and Sport}

Given the pervasiveness of stress in sport and the negative consequences of elevated stress on performance, stress appraisal and the ability to cope effectively with stressors are very important. As has been previously discussed, the COPE and Brief COPE are reliable and commonly used measures of coping in non-sport populations (Carver, 1997; Carver et al., 1989; Cooper, Katona \& Livingston, 2008; Kasi et al., 2012; Yusoff, Low \& Yip, 2010). Modified versions of the COPE have also been used to assess coping in sport (Crocker, Kowalski \& Graham, 1998). Crocker and Graham (1995) used nine of the original COPE items with slight modifications to make them relevant to sport, and added three additional sport-specific scales of coping to create a modified version of the COPE: active coping, seeking social support for instrumental reasons, planning, seeking social support for emotional reasons, denial, humor, 
behavioral disengagement, venting of emotion, suppression of competing activities, self-blame, wishful thinking and increasing effort. In a sample of competitive athletes $(n=337)$ (competing at the junior national, national, provincial, regional and university level) Crocker and Graham (1995) found that means were highest for the increasing effort, self-blame and active coping scales. Internal consistency for all subscales was found to be between 0.64 and 0.92 except for denial (0.42). Using the same modified COPE scale as Crocker and Graham (1995), a study of Australian athletes $(n=622)$ who competed at varying levels (recreational to international) found that means were highest for increasing effort, active coping, self-blame and planning. Overall, it seemed that athletes engaged more often in problem-focused coping strategies because those strategies allowed the athlete to manage performance-related challenges (Crocker et al., 1998).

Another modified version of the COPE was used to assess coping styles in Indonesian and Australian athletes $(n=283)$ (competing at local, regional, state/provincial levels) (Hoedaya \& Anshel, 2003). Additional items were added to the original COPE to assess effectiveness of choice of coping style adopted in response to a stressor. The cross-cultural nature of this study allowed for conclusions to be drawn about coping styles across cultures as well as selection of different coping strategies (problem-focused, emotion-focused, or "less useful" coping strategies) in response to varying stressors. Results indicated that selection of coping strategy differed dependent on culture (Australian vs. Indonesian) as well as the type of stressor. For example, the emotion-focused coping strategy of acceptance was used more often by Indonesian athletes when the stressor involved "making an error or failing to meet self-expectations" whereas Australian athletes were more likely than Indonesian athletes to use acceptance if the stressor involved an "injury or a team-mate getting dismissed". Furthermore, Indonesian athletes were more like to use the problem-focused coping strategy of active coping following a stressor 
such as "a very important game", whereas Australian athletes were more likely to use active coping following the stressor "doubting own performance". Overall, cultural differences were found in the use of active coping, venting emotions, acceptance and restraint. This study emphasized the importance that cultural differences may play in the appraisal of stress as well as the coping strategy employed by the athlete (Hoedaya \& Anshel, 2003). Furthermore, it provided evidence for the transaction approach to coping (Lazarus \& Folkman, 1984).

Several sport-specific measures have also been developed to assess coping in athletes. The Psychological Skills Inventory in Sport (PSIS R-5; Mahoney et al., 1987) is a 45 item scale that measures six psychological coping skills: Team Focus, Motivation, Metal Preparation, Anxiety Control, Confidence, and Concentration. However, the PSIS R-5 has been found to have poor factorial validity (Chartrand, Jowdy \& Danish, 1992). The Athletic Coping Skills Inventory (ACSI-28; Smith et al., 1995) is a 28-item survey that contains seven sport-specific subscales assessing coping: coping with adversity, peaking under pressure, goal setting, mental preparation, concentration, freedom of worry, confidence and achievement against coachability. A four-point Likert scale is used $(0=$ not at all to $3=$ very much so). Scores from each subscale are summed to yield a Personal Coping Resources score (scores range from 0 to 84). A positive coping score can also be found by adding the ratings from coping with adversity, peaking under pressure, goal setting and mental preparation, while a negative coping score can be found by summing the scores from confidence and achievement motivation, coachability, and freedom from worry. Internal consistency and test-re-test reliability coefficients have been found to be 0.86 and 0.87 respectively (Smith et al., 1995). Although the ACSI-28 is considered a good scale because it was designed specifically for athletes, the ACSI-28 has been criticized for several reasons: 1) it was not developed using coping processes as the theoretical foundation, 2) the 
ACSI-28 assesses effectiveness of the coping strategy employed by the athlete rather than "choice" of coping strategy, and 3) the ACSI-28 measures psychological skills and not specifically "coping" skills, which raises arguments about the conceptual difference between coping skills and psychological skills (Crocker, Kowalski \& Graham, 1998).

Anshel and Gangyan (2008) studied stress and coping strategies in elite athletes $(n=391)$ (national and international level) in the Peoples Republic of China. Eight different sources of acute stress were examined: making an error, being reprimanded by the coach, observing an opponent cheat, injury, bad call by an official, good performance by the opponent, spectator abuse, and bad performance due to weather conditions. The Coping Style in Sport Inventory (CSSI-C i.e. the Chinese version) was used to assess approach and avoidance coping styles in response to each of the eight stressors. Results indicated that the two coping styles (approach vs. avoidance) were more prevalent with certain stressors than others. For example, athletes were more likely to adopt avoidance coping strategies if the stressor was outside of their control e.g. coach reprimand (Anshel \& Gangyan, 2008). Overall, the results suggest that Lazarus and Folkman's (1984) transactional approach to coping is highly applicable to the athletic situation.

Nicholls, Polman and Levy (2012) examined pre-competition stress appraisal, emotions, post-competition coping and performance satisfaction in athletes $(n=557)$ who competed at varying levels of sport (international, county, club/university and beginner). Path analyses revealed that Lazarus' model (1984) of stress, appraisal and coping was an acceptable fit, supporting the association that exists between the three constructs. Findings indicated that perceptions of controllability were associated with stress appraisal. That is, controllable factors were appraised as challenges whereas uncontrollable factors were perceived as threats. Challenge appraisals were in turn associated with positive emotions and higher subjective performance 
satisfaction while threat relational appraisals were associated with unpleasant emotions and lower subjective performance satisfaction. Importantly, positive emotions indirectly impacted performance satisfaction through the use of task-focused coping. Task-focused coping was defined as any attempt made by the athlete to reduce stress through strategies such as mental imagery, relaxation, and effort expenditure. In turn, unpleasant emotions impacted performance satisfaction through the use of disengagement (withdrawal and venting of emotions) and distraction-oriented (e.g. mental distraction and distancing) coping. Findings from this study have several important implications. Firstly, the role of appraisal of "threat" vs. "challenge" is crucial in the experience of stressful events for athletes as previously indicated by Hanton et al. (2012) and Neil et al. (2011). Secondly, as outlined by Lazarus (1966), it is also important to study the role of emotions (as well as coping) in stressful events for athletes. Lastly, different forms of coping are adopted when a situation is appraised as either controllable or uncontrollable i.e. a transactional approach to coping. This is similar to findings from Roth and Cohen (1986) with regards to approach and avoidance coping strategies. That is, Nicholls, Polman and Levy (2012) found that task-focused coping strategies are employed when a challenge appraisal is made from a controllable stressor, while distraction or disengagement coping strategies are employed when a threat appraisal is made from an uncontrollable stressor.

\section{Intermittent Conclusions}

Overall, the stress and coping literature emphasizes the importance of appraisal in the stress, appraisal and coping model (Lazarus \& Folkman, 1984). An event must be appraised as "meaningful" in order for it to be stressful. Furthermore, emotions are also important in the appraisal process because they signify what is important to the person (Lazarus, 1966). The coping process is approached from either a transactional or a trait theoretical framework where 
the former highlights the situation-specific nature of coping strategies and the latter outlines the rigidity and dispositional characteristic of coping styles (Carver, Scheier \& Weintraub, 1989; Lazarus \& Folkman, 1984; Parkes, 1986). Differentiations are also made between problemfocused coping vs. emotion-focused coping (Carver et al., 1989), and approach vs. avoidant coping (Roth \& Cohen, 1986). Within the sport psychology literature, findings from Hanton, Wagstaff and Fletcher (2012), Neil et al. (2011) and Nicholls, Polman and Levy (2012) supported the idea that appraisal of "challenge vs. threat" and controllability of a stressful event impact the types of emotions experienced by the athlete as well as the coping strategy they adopt. Anshel and Gangyan (2008) provided support for the use of different coping styles (approach vs. avoidance) dependent on the type of stressor i.e. transactional theory. Lastly, Hoedaya and Anshel (2003) studied cultural differences and athletes' choice of coping style in response to varying stressors. Hoedaya and Anshel provided support for the situation-specific adoption of problem-focused vs. emotion-focused coping strategies across cultures. Future directions may include explorations into psychological constructs that could positively impact stress appraisal and coping processes in athletes and therefore serve as effective stress-management tools.

\section{STRESS, COPING AND INJURY IN SPORT}

\section{Prevalence of Injury in Sport}

In an epidemiological study of collegiate athletes and injury prevalence, Hootman, Dick and Agel (2007) collected injury data from the National Collegiate Athletic Association (NCAA) Injury Surveillance System (ISS) across 15 sports between the 1988-1989 and 2003-2004 seasons. As defined by the ISS, injury had to be sustained during participation in collegiate practice or competition, required medical attention and resulted in at least one day of absence from athletic participation. Over the time span of 16 years, rates of injury averaged out to 1 
injury every 2 games and 1 injury every 5 practices for a team of 50 athletes. Findings indicated that the highest game injury rates occurred during the in-season (as compared to pre or post season rates) and in Division I athletes (as compared to Division II and III athletes) with 58\% of game injuries occurring due to contact with another player. Rate differences in types of injuries indicated that ankle injuries accounted for a quarter of the injuries at a rate of 11,000 per year and primarily in men's basketball and football. Anterior cruciate ligament (ACL) injuries occurred at a rate of 2, 000 per year and were highest in number in football and highest in rate in women's gymnastics. Concussions occurred at a rate of 3, 753 per year and were also highest in number in football but highest in rate in women's ice hockey. Across the 16 year time span, ankle sprain injuries remained stable in rate while both concussions and ACL injuries increased in rate. Recommendations were made to explore and understand all causative sources of athletic injury and to develop comprehensive injury prevention initiatives.

Findings from a range of other studies indicate that injury rates vary dependent on athletic population as well as sport. Hanson, McCullagh and Tonymon (1992) reported that $66.3 \%$ of Division I and II track and field athletes $(n=181)$ sustained at least one injury over the course of a season. In a sample of junior college (NJCAA) Division I and II female softball players ( $n=108), 52$ of 108 athletes reported at least one injury (Laws-Gallien, 2001). Johnson and Ivarsson (2011) found that 39\% of high school soccer players $(n=108)$ missed at least one practice due to injury (Johnson \& Ivarsson, 2011). Across a sample of Division IA football players, each player missed an average of 5 days of practice/competition due to injury (Petrie, 1993). Stephen, Pensgaard and Bahr (2009) reported that in a sample of Swedish high school soccer players $(n=1430), 380$ injuries were recorded over the course of the 2005 season. Lastly, Burt and Overpeck (2001) indicated that $22 \%$ of emergency room visits for individuals aged 5 to 
24 years old were attributed to sport-related injuries. Furthermore, sport-related injuries (as compared to other injuries) were more likely to be to the head and the upper or lower extremities (Burt \& Overpeck, 2001).

\section{Stress and Injury in Sport}

Vulnerability for athletic injury is impacted by much more than just physical factors; it is the result of the interaction between internal psychological (e.g. coping responses, life event stress), internal biological (e.g. body composition), external physical (e.g. equipment) and external sociocultural (e.g. sport norms) factors (Wiese-Bjornstal, 2010). Although it is still considered to be a relatively young field, psychosocial factors that impact injury have been explored for the past couple of decades (Pargman, 1999; Redmond, 1996; Shamus, Kelleher \& Foran, 2001). Of these factors, stress and coping factors have been found to be some of the strongest predictors of athletic injury (Blackwell \& McCullagh, 1990; Shamus, Kelleher \& Foran, 2001).

To better understand the role of these factors in vulnerability to injury, Williams and Andersen (1998) developed a multicomponent model of psychological stress and injury. This model integrates the combined effects that physiological, psychological, behavioral, social and stress history may have on sport injury prediction (Pargman, 1999). More specifically, this model suggests that personality (i.e. locus of control, hardiness, competitive trait anxiety etc.), history of stressors (i.e. life event stress, daily hassles, injury history), and coping resources (i.e. coping style, social support, stress management etc.) will moderate the stress response to a potentially stressful situation (Johnson, 2007; Petrie, 1993; Williams \& Andersen, 1998). The stress response involves the interaction between the cognitive appraisal of the stressor as well as the physiological and attentional changes, which are in turn associated with vulnerability to injury. 
In this model life event stress is defined as the distress associated with major life events such as the breakup of a relationship or starting at a new school while daily hassles stress is defined as the strain associated with minor daily irritations or problems such as getting stuck in traffic or being late (Williams \& Andersen, 1998).

Essentially this model states that the amount of stress that an athlete will face in any athletic situation will depend on their appraisal of the stressor as a threat. This is consistent with stress and coping literature (Lazarus, 1966; Lazarus \& Folkman, 1984; Nicholls, Polman \& Levy, 2012). The athlete will then engage their coping skills and either successfully overcome the "challenge" by relaxing and having confidence in their abilities, or they will perceive the situation as a "threat" and become excessively physically aroused (Shamus, Kelleher \& Foran; 2001, Williams \& Andersen, 1998). This increase in state anxiety will cause increased muscle tension as well as narrowing of peripheral attention. Increased muscle tension is associated with greater risk for injury due to decreased coordination and increased impulsivity (Johnson, 2007). Narrowing of peripheral vision can impair the athlete from picking up on external cues that would normally signal physical harm (Shamus, Kelleher \& Foran; 2001, Williams \& Andersen, 1998).

Since the first study investigating the link between psychological stress and injury in football players was conducted by Holmes in 1970 and since the development of Williams and Andersen's (1988) stress-injury model, a number of studies have since further strengthened the evidence for this association (Steffen, Pensgaard, \& Bahr, 2008; Williams \& Andersen, 1998). Both qualitative and intervention studies provide support for the stress-injury model (Johnson, 2007). Hanson, McCullagh and Tonymon (1992) studied stress and injury in male and female Division I and II track and field athletes $(n=181)$ and found that as negative life stress 
increased, severity of the injury increased. However, frequency of injury did not increase with increased negative life stress. Rather, positive life stress was associated with increased frequency of injury (Hanson et al., 1992). In a study of male collegiate Division I A football players ( $n=$ 158), $60 \%$ of injury variance could be explained by positive life stress, competitive anxiety and coping skills for athletes who were starters (Petrie, 1993). However, for non-starters, there was no association between psychosocial variables and injury (Petrie, 1993). Laws-Gallien (2001) found that negative life stress was significantly different between injured and non-injured athletes in a sample of female junior college (NJCAA) Division I and II softball players ( $n=$ 108) aged 18-21 (Laws-Gallien, 2001). Furthermore, Johnson and Ivarsson (2011) found that negative life stress accounted for $7 \%$ of variance in injury incidence in a sample of Swedish high school soccer players $(n=108)$. This study provided further support for Williams and Andersen's (1998) stress-injury model given that 5 predictors (negative life event stress, stress sensitivity, negative coping, somatic trait anxiety, and mistrust) collectively accounted for $23 \%$ of variance in injury status (Johnson \& Ivarsson, 2011). However, senior high school soccer players experience different age-related stressors than collegiate athletes (Wylleman \& Lavallee, 2004) which may result in a different stress-injury relationship in these two separate populations.

Although the majority of studies support the association between high life stress (especially negative life stress) and increased injury susceptibility (Johnson, 2007), some studies have found no association (Ivarsson \& Johnson, 2010) while others have pointed to the important of positive stress in injury risk (Hanson et al., 1992; Petrie, 1993). Further exploration is therefore warranted especially within collegiate athletes who face unique stressors (Etzel; 2009; Lu et al., 2012). Several recommendations have been made for future studies: 1) explore which specific variables contribute to risk for athletic injury within a range of diverse athletic 
populations (Petrie, 1993), 2) develop sport-specific scales to assess psychological variables that contribute to risk for athletic injury (Hanson, McCullagh \& Tonymon, 1992), and 3) use a multivariable approach to predict injury incidence because individual predictors do not provide a complete picture (Hanson, McCullagh \& Tonymon, 1992).

Research has also further addressed the moderating variables included in Williams and Andersen's (1998) stress-injury model. That is, studies have explored coping resources such as social support and coping behaviors (Johnson, 2007; Wiese-Bjornstal, 2010). Hanson, McCullagh and Tonymon (1992) found that in a sample of Division I and II track and field athletes $(n=181)$, coping resources predicted frequency and severity of injuries. In fact, across three groups of athletes (no injuries, one injury and more than one injury), coping resources was found to discriminate best between frequency of injury with positive life stress as the second strongest discriminator. Furthermore, coping resources were also the strongest discriminator of severity of injury, followed by negative life stress. Coping was assessed using a modified version of the Coping Resource Section of the Stress Audit Questionnaire. Smith, Smoll and Ptacek (1990) provided support for the combined effects of coping behaviors and social support on prediction of athletic injury. That is, only athletes low in both social support and coping strategies showed increased vulnerability to athletic injury. In a study of female collegiate gymnasts, when athletes had low social support, negative life event stress accounted for $11 \%$ to $22 \%$ of injury variance while positive life stress accounted for $14-20 \%$ of injury variance (Petrie, 1992). Petrie (1993) further studied the impact of moderating variables on the stress-injury relationship in a sample of NCAA Division 1A football players $(n=153)$. Athletes completed measures of coping, competitive trait anxiety and life stress at the team's first meeting of the season. To assess coping skills, the Athletic Coping Skills Inventory was used, which assesses 
three types of coping strategies i.e. emotional, cognitive and behavioral. At the end of the season coaches provided injury information on each player which included the number of practice days and competitive games they missed due to injury (i.e. injury was coded as 'time loss'). Results indicated that for athletes who were starters, $60 \%$ of injury variance could be explained by positive life stress, competitive anxiety and coping skills indicating the importance of the inclusion of coping skills within the stress-injury model.

More recently, Johnson and Ivarsson (2011) studied psychological predictors of athletic injury among male and female high school soccer players $(n=108)$ in Sweden. They found no difference in coping resources (as measured by the ACSI-28) between injured and non-injured athletes. They did find that $23 \%$ of injury variance could be explained by the interaction between negative life event stress, negative coping, somatic trait anxiety, mistrust and stress susceptibility. Johnson and Ivarsson (2011) concluded that there was partial support for Williams and Andersen's (1998) stress-injury model. From the research outlined here, there is some research that supports the moderating role that social support and coping behaviors play in Wiliams and Andersen's stress-injury model (Johnson, 2007). However, none of the research specifically addresses coping styles such as emotion-focused vs. problem-focused or approach vs. avoidant coping which are forms of coping that the stress research details to be important to consider (Folkman \& Lazarus, 1980; Roth \& Cohen, 2012). Furthermore, they have not explored aspects of appraisal i.e. challenge vs. threat and controllability of the stressful event that have been shown to impact the coping strategy the athlete adopts (Nicholls, Polman, \& Levy, 2012) which may also affect their vulnerability for injury. Additionally, there has been no study as of yet that explores the role that self-compassion may play as a coping resource in the stress-injury model. 


\section{Stress Management for Injury Prevention}

Within the sport psychology literature, stress management techniques have been supported as important avenues for injury prevention in athletes (Wiese-Bjornstal, 2010). In a randomized clinical trial, Perna et al. (2003) explored the effect that a cognitive behavioral stress management (CBSM) would have on the incidence of injury and illness in male and female junior varsity collegiate rowers $(n=40)$. Rowers in the CBSM group received 7 sessions of stress inoculation training followed by stress management skills (relaxation strategies), experiential exercises and homework assignments. Findings indicated that the rowers in the CBSM group had significantly fewer days missed due to injury or illness than the rowers in the control group. The positive impact of CBSM techniques on injury have been replicated in junior elite soccer players (Edvarsson, Ivarsson \& Johnson, 2012) and rugby players (Maddison \& Prapavessis, 2005) while other relaxation and stress management techniques have also had significant impacts on injury reduction (Johnson, Ekengren \& Andersen, 2005). Mindfulness has also emerged as a promising injury prevention technique due to its impact on stress appraisal (Weinstein, Brown \& Ryan, 2009). In a study of male junior elite high school soccer players $(n=$ 42 ) in Sweden, it was found that $67 \%$ of the athletes who were randomly assigned to the mindfulness-based program did not experience injury over the course of the season while only $40 \%$ of the players in the attentional control group remained injury-free (Ivarsson et al., 2015). Given the impact that narrowing of peripheral vision and attention has on injury risk i.e. stress response (Williams \& Andersen, 1998), it was concluded that mindfulness is a viable option for psychological interventions for injury prevention. It is evident from these studies that stress management and mindfulness techniques contribute to lower injury incidence. Therefore, further explorations into alternative stress management and mindfulness techniques are warranted. 
Intermittent Conclusions

Stress and coping factors have been linked to heightened risk for athletic injury (Blackwell \& McCullagh, 1990; Shamus, Kelleher \& Foran, 2001). Studies across a wide range of sports and athletic populations as well as qualitative and intervention studies provide support for the stress-injury association (Johnson, 2007). Specifically, higher life stress has consistently been found to predict increased susceptibility to injury in male and female high school soccer players (Johnson \& Ivarsson, 2011; Stephen, Pensgaard \& Bahr, 2008), junior college (NJCAA) Division I and II female softball players (Laws-Gallien, 2001), collegiate Division I and II track and field athletes (Hanson, McCullagh \& Tonymon , 1992), and collegiate Division 1A football players (Petrie, 1993). Furthermore, evidence indicates that low coping resources alongside high life stress further exacerbates the stress injury relationship (Johnson, 2007). That is, collegiate athletes who suffer from more frequent injuries have significantly fewer coping resources or engage in more dysfunctional coping strategies (Carver, 1997) than non-injured athletes (Hanson, McCullagh \& Tonymon , 1992; Ivarsson \& Johnson, 2010; Petrie, 1992; Petrie, 1993). Although the majority of studies support the association between high life stress (especially negative life stress) and increased injury susceptibility (Johnson, 2007), some studies have found no association (Ivarsson \& Johnson, 2010) while others have pointed to the importance of positive stress in injury risk (Hanson, McCullagh \& Tonymon , 1992; Petrie, 1993). Additionally, recommendations have been made to further consolidate the stress-injury association in a wider range of athletic populations (Petrie, 1993) given that high school soccer players and professional athletes will experience different age-related stressors than collegiate athletes (Wylleman \& Lavallee, 2004). Lastly, it has been strongly suggested that a multivariable 
approach be used to predict injury incidence because individual predictors do not provide a complete picture of injury risk (Petrie, 1993).

Andersen and William's (1988) stress-injury model is to date the most influential multivariable approach to predicting athletic injury (Johnson, 2007). There is considerable research that supports the moderating role of coping resources such as social support and stress management strategies (Hanson et al., 1992; Johnson, 2007; Johnson \& Ivarsson, 2011; Petrie, 1993; Smith, Smoll \& Ptacek, 1990; Wiese-Bjornstal, 2010). However, none of the research specifically addresses coping styles such as emotion-focused vs. problem-focused or approach vs. avoidant coping which are forms of coping that the stress research details to be important to consider (Folkman \& Lazarus, 1980; Roth \& Cohen, 2012). Furthermore, they have not explored aspects of appraisal i.e. challenge vs. threat and controllability of the stressful event that have been shown to impact the coping strategy the athlete adopts (Nicholls, Polman, \& Levy, 2012) which may also affect their vulnerability for injury.

Sustaining an injury at the collegiate level can cause lengthy absences from practice/competition as well as emotional distress (e.g. depression, anxiety, fear of re-injury and self-esteem issues) (Kvist, Sporrstedt, \& Good, 2005; Leddy, Lambert \& Ogles, 1994). For this reason, stress management skills and coping strategies are important to develop and explore because they can help buffer the impact of stress on the athlete and thereby potentially reduce the risk for athletic injury (Johnson, 2007; Williams \& Andersen, 1998). Cognitive behavioral stress management strategies primarily reduce stress through lowering of cognitive appraisal of stress and increasing control expectancies (Gaab et al., 2003). This is consistent with Lazarus (1966) theory of psychological stress and coping. The inclusion of appraisal in Lazarus and Folkman's (1984) model dictates that the focus of coping should not be on the stressor but rather on the 
individual's appraisal of the stressor, which is similar to the emphasis placed on stress appraisal in Williams and Andersen's (1998) stress-injury model. Therefore, stress management strategies or coping resources that have an appraisal component may be useful to further explore within the context of stress and injury in collegiate athletes. One coping resource that has not yet been explored in the stress-injury model is self-compassion.

\section{SELF-COMPASSION, STRESS AND COPING}

Psychological variables play an integral role in buffering the impact of stress and negative life events on youth. Resilience studies indicate that psychological factors such as selfesteem and coping style may play a protective role in the experience of stress (Dumont \& Provost, 19999; Grant et al., 2000; Seiffge-Krenke, 2000). As has been previously outlined, stress occurs when an individual appraises the demands of a situation to be higher than their coping abilities (Lazarus, 1966). Self-compassion may be adaptive for positive psychological functioning and well-being in the face of stress (Neely et al., 2009; Neff, 2003b; Neff, Kirkpatrick \& Rude, 2007b; Allen \& Leary, 2010). Self-compassion contributes to the appraisal process by changing the experience of self-evaluative appraisals (Neff, 2003a; Neff et al., 2007a; Mosewich et al., 2011).

Leary et al. (2007) studied self-compassion in the face of unpleasant events that have high self-relevance. They aimed to assess both the cognitive and emotional processes used by individuals high in self-compassion to cope with negative events in their daily lives, unpleasant hypothetical scenarios, interpersonal feedback as well as negative personal experiences (Leary et al., 2007). In Study 1, Leary et al. (2007) examined daily hassles in undergraduate students at four-time points over the period of three weeks. Results indicated that the 'common humanity' items of the Self-Compassion Scale most strongly predicted positive reactions to daily stressors 
suggesting that self-compassion can be used as a healthy resource by helping individuals reframe their stressors as events that are a part of life and no more difficult for them than it is for anyone else. In Study 2, participants responded to three unpleasant hypothetical scenarios of failure, humiliation and loss. Results were similar to Study 1 and indicated that self-compassion predicts more positive responses to hypothetical scenarios as well. Specifically, self-compassion predicted cognitive and emotional re-appraisal of unpleasant as indicated by less catastrophizing, greater balance of emotions and less personalization. Study 3 was unique because it addressed the use of self-compassion as a resource for unpleasant interpersonal feedback. Using one-way video interactions, participants introduced themselves and spoke about hobbies, likes, dislikes, etc. for three minutes. The participant was then given an envelope containing feedback about their introduction from the "observer" on the other side of the video interaction. Participants were randomly assigned to either receive positive feedback (5's, 6's and 7's) or neutral feedback (3's, 4's and 5's). This study specifically assessed the participants' appraisal for feedback. Results found that individuals high in self-compassion were more likely to make similar attributions for either positive or neutral feedback while individuals low in self-compassion were more likely to attribute neutral feedback less to themselves and positive feedback more to themselves. This makes sense in light of the mindful acceptance and common humanity components of self-compassion that allow individuals to view both successes and failures as a part of life and maintain psychological distance from unpleasant self-relevant emotions (Leary et al., 2007). Lazarus' stress model emphasizes the overlap that exists between stress and emotions. In fact, Lazarus argues that a deeper understanding of the antecedents of emotions and the processes of emotions will shed light on what is important to people and what causes stress 
(Lazarus, 1966). Lazarus' model suggests that self-compassion may reduce stress due to its effect on emotional balance.

Interestingly, Leary et al. (2007) also examined whether there were differences in the effects of self-compassion when it was experimentally induced versus trait self-compassion. Self-compassion was experimentally induced by asking participants to respond to three writing prompts that focused on each component of self-compassion. Findings indicated that individuals in the self-compassion induction were more likely to appraise the negative event as something that was caused by their personal characteristics while at the same time reporting lower negative affect. Furthermore, individuals low in trait self-compassion were positively impacted by the self-compassion induction while individuals high in trait self-compassion were not at all affected by the induction. These findings suggest that both an induction of self-compassion as well as trait self-compassion serve to promote acceptance of personal responsibility for actions while also reducing distress and negative emotions. As a whole, Leary et al. (2007) contributed to a greater understanding of the role of self-compassion to cope with both daily stressors as well as negative life experiences. Furthermore, self-compassion played a role in reactions to interpersonal feedback, which may be specifically relevant for athletes who are constantly receiving criticism or unpleasant feedback about performances (Leary et al., 2007).

Self-compassion has also been directly examined in relation to coping strategies. In fact, in Neff's (2003a) earliest research on self-compassion she argues that self-compassion is a form of "emotional approach" coping (as opposed to emotional avoidance coping) that allows individuals to identify and express their emotions in a balanced and psychological adaptive way. Allen and Leary (2010) examined the role that self-compassion plays in the experience of negative life events and coping. Their review suggested that while avoidance-oriented coping 
strategies have been negatively related with psychological well-being and are therefore maladaptive, self-compassion is also negatively related with avoidance-coping strategies. That is, individuals high in self-compassion are more likely to accept responsibility for the mistakes yet treat themselves with kindness in the face of these failures. Perhaps even more importantly, selfcompassionate individuals tend to want the best for themselves which naturally leads to positive self-changes (Allen \& Leary, 2010). This may be especially relevant for athletes who inevitably face setbacks such as poor performance or defeat in a competition (Mosewich, Crocker \& Kowalski, 2013). Thus, highly self-compassionate athletes may display a greater desire to seek ways to improve which may in turn lead them to continue to practice hard and exert effort despite the setback.

Further studies of self-compassion and coping strategies have also addressed the link between self-compassion, perceived competence, academic achievement goals, fear of failure and intrinsic motivation in the face of academic failure (Neff, Hsieh \& Dejitterat, 2005). Undergraduate students were recruited for two separate studies ( $n=222$ and $n=110)$. Analyses focused on the students who were unsatisfied with their recent mid-term grades i.e. perceived academic failure. Firstly, results indicated that self-compassion was significantly positively associated with mastery goals, intrinsic motivation, and perceived competence and negatively associated with performance-avoidance goals. This suggests that self-compassion plays a positive role in motivating students to continue developing their skills despite perceived failure. Furthermore, self-compassion was positively associated with the emotion-focused coping strategies of growth and acceptance as well as positive re-interpretation while it was negatively associated with the emotion-focused strategy of venting of emotions. With regard to avoidancecoping strategies, self-compassion was negatively associated with denial and mental 
disengagement. Lastly, self-compassion showed no association with any of the five problemfocused strategies. These results suggest that self-compassion plays its biggest role in coping by promoting emotional clarity and equanimity, as well as an accepting mindset i.e. emotionfocused coping. This may, however, help the individual to then proceed to take steps to reduce the causes of stress i.e. problem-focused coping (Neff, Hsieh \& Dejitterat, 2005).

There are currently only two measures that assess self-compassion: the 26-item selfcompassion scale (SCS; Neff, 2003a) and the 12-item Self-Compassion Scale-Short Form (SCSSF; Raes et al., 2011). The SCS-SF assesses three different aspects of self-compassion: selfkindness (e.g., 'I try to be understanding and patient towards aspects of my personality I don't like’) versus self-judgment (e.g., "I'm disapproving and judgmental about my own flaws and inadequacies"); common humanity (e.g., "I try to see my failings as part of the human condition'”) versus isolation (e.g., "'When I'm feeling down, I tend to feel like most other people are probably happier than I am"); and mindfulness (e.g., "When something painful happens I try to take a balanced view of the situation'”) versus over-identification (e.g., "'When I'm feeling down I tend to obsess and fixate on everything that's wrong.'). A five-point Likert scale is used to rate each item on the subscales (1=almost never to $5=$ almost always). An overall selfcompassion score (scores range from 6 to 30) is obtained by reverse coding the negative items and summing up the mean scores on the six subscales. Scale validation research for the SCS-SF indicates that within German and English samples, all six subscales are highly inter-correlated and the SCS-SF demonstrates good internal consistency (Cronbach's alpha $\geq 0.86$ ) (Neff, 2003a; Neff et al., 2005). The SCS-SF has also been found to be nearly perfectly correlated to the long form SCS (r>.97) (Raes et al., 2011).

\section{Intermittent Conclusions:}


The aforementioned findings may be particularly relevant to athletic situations given the occurrence of self-evaluations and causal attributions after performance success or failure in competitive situations. Moreover, these findings suggest that self-compassion may serve as a buffer against negative self-evaluations after failure in sport. Given that negative self-evaluations are a source of stress in sport, and stress can be reduced through re-appraisal of the stressor (Lazarus \& Folkman, 1984; Park \& Folkman, 1997), self-compassion may enhance adaptive coping with stress. It has been found that self-compassionate individuals are better able to make accurate self-appraisals while maintaining balanced emotional awareness (Leary et al., 2007). Different coping styles also have an important impact on resolving stress (Carver et al., 1989; Lazarus \& Folkma, 1984). Emotion-focused coping uses positive re-interpretation of negative events to deal with the emotional distress associated with a stressful situation (Neff, Hsieh \& Dejitterat, 2005). Focusing on emotions may in fact allow the individual to re-appraise the stressor and come to a healthier attribution of the event (Stanton et al., 2000). Self-compassion is a form of emotional approach coping (Neff, 2003a) and has been linked with adaptive coping in the face of failure (Neff, Hsieh \& Dejitterat, 2005). Furthermore, self-compassion was positively associated with the emotion-focused coping strategies of growth and acceptance as well as positive re-interpretation while it was negatively associated with the emotion-focused strategy of venting of emotions (Neff, Hsieh \& Dejitterat, 2005). Self-compassionate individuals are more likely to accept responsibility for the mistakes yet treat themselves with kindness in the face of these failures (Allen \& Leary, 2010). Self-compassion is also negatively correlated with avoidance coping (Allen \& Leary, 2010); specifically, the avoidance coping strategies of denial and mental disengagement (Neff, Hsieh \& Dejitterat, 2005). The literature outside of the athletic context therefore suggests that there is a strong argument for the use of self-compassion as a 
coping resource for stress in athletes. This has yet to be studied in a formal manner. Furthermore, Lazarus and Folkman's (1984) eight underlying properties of stress have been found to be relevant to stress in the athletic context. However, studies have yet to address how selfcompassion may fit in that model and alter the experience of stress in athletes.

\section{SELF-COMPASSION INTERVENTIONS}

Self-compassion may be an appropriate psychological variable to study within the context of stress management and the stress-injury relationship because it is modifiable both through short-term interventions such as self-compassion writing inductions (Leary et al., 2007) and longer-term interventions such as mindfulness-based stress reduction (MSBR) (Chiesa \& Serretti, 2009), compassionate based therapy (CMT) (Gilbert \& Proctor, 2006) and loving kindness meditation (LKM) (Hofmann, Grossman \& Hinton, 2011). The following section will provide further detail of short-term and long-term self-compassion interventions.

\section{Short-term Interventions}

Self-compassion may be promoted through short-term interventions. Leary et al. (2007) attempted to induce a self-compassionate perspective in a sample of male and female undergraduate students $(n=115)$. Participants were asked to recall and write down all the details about a negative event from their past that had made them feel very badly about themselves. They were then randomly assigned to a self-compassion induction group, a self-esteem induction group, a writing group or a true control group. In the self-compassion induction group the participants were asked to respond to three prompts that addressed the negative event using the three components of self-compassion i.e. self-kindness, common humanity and balanced awareness. The results indicated that individuals higher in self-compassion were more likely to take responsibility for the negative event while at the same time not feel any distress or negative 
affect associated with this attribution. Thus, individuals higher in self-compassion demonstrated more realistic self-appraisals. Interestingly, the self-compassion induction did not affect individuals who were already high in self-compassion; it solely increased self-compassion in those individuals low in self-compassion.

\section{Long-term Interventions}

Self-compassion may also be promoted through more sustained interventions. Neff et al. (2007a) conducted a study to investigate changes in self-compassion over the course of one month. A Gestalt two-chair exercise was used to challenge existing maladaptive and self-critical beliefs in counseling session over the course of a month with 40 undergraduate students. The counselor who facilitated the Gestalt exercise rated levels of self-compassion shown in the participants' responses during the exercise. Results indicated that self-compassion levels significantly increased over the one-month interval and were associated with improvements in well-being. Improved well-being included decreased self-criticism, rumination and anxiety. Neff et al.'s (2007a) study suggested that self-compassion is a modifiable construct and therefore targetable by interventions. The results of this study have important implications for the use of self-compassion in interventions for athletes.

Mosewich et al. (2013) designed a week-long (which is considered long-term in comparison to a brief writing induction) psychoeducational intervention for female varsity athletes who had self-identified as high in self-criticism. Participants were randomly assigned to either a self-compassion intervention group or an attention control group. The seven day intervention included an initial session composed of a psychoeducational presentation (i.e. basic information about stress, coping strategies and self-compassion using empirical research findings), an applied writing task to practice self-compassion, the completion of a set of 
questionnaires, and several modules designed to build self-compassionate skills to be completed during the seven-day period. Each module was essentially a variation of the same applied writing exercise where athletes were first asked to recall and write down a negative event they had experienced in sport over the past week, and then asked to respond to the recalled scenario using different self-compassion skills. To promote 'common humanity', athletes were asked to think of ways that other athletes may experience similar events. Similarly, to promote 'self-kindness' athletes were asked to write a letter expressing kindness and understanding to a close friend who was experiencing the same negative event. Lastly, athletes were asked to re-write the scenario in an objective and unemotional manner to promote 'mindfulness' and 'balanced awareness'. The athletes completed a second set of questionnaires one week after the intervention. This time frame was chosen to give the athletes time to internalize some of the skills and try to apply them within the sporting context. Results indicated that the intervention was successful in decreasing self-criticism and concern over mistakes, and increasing self-compassion over the time-period of a week. This finding was important to indicate maintenance of self-compassion promotion over time. It was concluded that self-compassion interventions can be useful to teach athletes how to cope with negative events in a manner that reduces self-criticism (Mosewich et al., 2013). Selfcriticism and dealing with difficult sporting situations are significant sources of stress for collegiate athletes given their high pressure environment that collegiate athletes. This study was important in illustrating that elements of self-compassion can be taught to athletes to help them deal with negative events i.e. as a coping resource and mental skill.

Self-compassion may also be increased through therapeutic approaches such as mindfulness and compassion-based psychotherapy (Neff, 2011). Mindfulness-Based Stress Reduction (MBSR) is a group-based program that uses mindfulness meditation to heighten 
awareness, reduce stress and negative affect, improve coping with distress and increase selfcompassion (Birnie, Speca \& Carlson, 2010; Chiesa \& Serretti, 2009; Grossman et al., 2004). At the individual level intervention, mindfulness can also be used to reduce anxiety and stress by allowing thoughts and feelings to flow freely in and out of the mind with understanding and kindness and without judgment (Kabat-Zinn, 1991). Compassionate Mind Training (CMT) is a form of group therapy that uses acceptance and compassionate thoughts to combat self-criticism (Gilbert \& Proctor, 2006). It has been found to increase warmth and reassurance towards the self and decrease anxiety, depression and self-criticism (Allen \& Leary, 2010). Additionally, meditation practices that are based on self-compassion and unconditional positive regard, such as loving-kindness meditation (LKM), may also be used to enhance well-being (Hofmann, Grossman \& Hinton, 2011). LKM is associated with decreases in mood disturbance, increases in positive affect as well as emotional processing and empathy. Overall, psychotherapeutic approaches such as MBSR (Birnie, Speca \& Carlson, 2010; Chiesa \& Serretti, 2009; Grossman et al., 2004), CMT (Gilbert \& Proctor, 2006) and LKM (Hofmann, Grossman \& Hinton, 2011) may be potential resources for long-term development of self-compassion.

\section{SELF-COMPASSION AND SPORT}

Only a handful of studies exist that directly examine self-compassion in highperformance athletes. In the related exercise context, self-compassion has been directly studied in a sample of female exercisers $(n=252)$ (Magnus, Kowalski \& McHugh, 2010). Results supported a significant association between self-compassion and well-being in measures of intrinsic motivation to exercise and social physique anxiety. It was proposed that selfcompassion may be an important resource for women in terms of promoting feelings of selfworth and lowering self-evaluation (Magnus, Kowalski \& McHugh, 2010). Self-compassion was 
studied in a similar manner as a potential resource for female high school athletes $(N=151)$ (Mosewich et al, 2011). Mosewich et al. explored the relationship between self-compassion, selfconscious emotions and unhealthy self-evaluative thoughts and behaviors. Given the role that self-compassion plays in promoting self-kindness, balancing emotions and reducing overidentification with negative emotions, it was hypothesized that self-compassion may prove to serve as an important emotion regulation strategy in female athletes. Furthermore, selfcompassion may be an important buffer against self-evaluative thoughts by changing the experience of self-evaluative attributions in sport. Athletes completed a set of questionnaires assessing self-compassion, self-conscious emotions, social physique anxiety, fear of failure and fear of negative evaluation (Mosewich et al., 2011). Every participant was involved in at least one sport over the course of the preceding 12 months, with the majority involved in at least two sports. Results found that self-compassion was negatively correlated with shame-proneness, social physique anxiety, fear of failure and fear of negative evaluation. Findings support the utility of self-compassion in self-evaluative processes that may be unhealthy for female athletes. Further exploration into the role of self-compassion in sport-specific situations such as failure events or important competition losses is warranted (Mosewich et al., 2011). In addition, evidence for the utility of self-compassion in male athletes is needed.

Reis et al. (2015) studied the importance of self-compassion in sport situations that are emotionally difficult. One hundred and three women aged between 14 and 25 who had taken part in organized sport within the past year participated in the study. After completing measures of self-compassion, participants were asked what their thoughts and emotions would be in response to a hypothetical scenario in which they were responsible for their team's loss. They were also asked to recall the worst thing that had happened to them in their sport over the past year and 
state what thoughts and emotions they experienced. Overall, findings supported the positive role that self-compassion plays in healthy responses to emotionally difficult situations. In response to the hypothetical scenario, self-compassion was negatively correlated with catastrophizing and negative affect, and positively associated with equanimous thoughts and behaviors. For the recalled scenario, self-compassion predicted lower anxiety, sadness and self-conscious emotions, and was positively associated with positive thoughts and reactions such as "I tried to be kind to myself" and "I tried to give myself time to come to terms with it". Additionally, self-compassion was negatively correlated with reactions such as "I was really hard on myself", "I'm a loser" and "I seem to have bigger problems than others". Interestingly, although this study included a second phase where participants were randomly assigned to either a self-compassion, self-esteem or writing induction and then asked to respond to the same hypothetical scenario, all findings were non-significant in the induction phase. The findings from phase II were unexpected given that several other studies have found positive impacts of self-compassion inductions on failure experiences and unpleasant self-relevant events (Breines \& Chen, 2012; Leary et al, 2007). Reis et al. (2015) concluded that female athletes higher in trait self-compassion respond in healthier ways to hypothetical and recalled emotionally difficult sport scenarios than athletes lower in selfcompassion. However, as was mentioned with the previous two studies, male athletes were not included within the sample population.

\section{Special Considerations}

Although self-compassion research indicates that the potential benefits of selfcompassion make it a very promising avenue for athletes to pursue, some athletes have expressed concern that self-compassion may promote passivity and acceptance of mediocrity (Ferguson et al., 2014; Reis et al., 2015; Sutherland et al., 2014). Perhaps this helps explain why there has yet 
to be any research on self-compassion in male athletes. However, self-compassion has been linked to the opposite of acceptance of mediocrity i.e. self-improvement motivation and mastery goals (Breines \& Chen, 2012; Neff, Hsieh, \& Dejitterat, 2005). Breines and Chen (2012) explored the relation between self-compassion after making a mistake and self-improvement motivation in three separate studies of male and female undergraduate students $(N=69, N=100$, $N=103)$ and one study of adults $(N=78)$. Four important findings were made in separate experiments that briefly induced self-compassion. First, self-compassion was associated with more incremental beliefs of self-improvement. That is, individuals higher in self-compassion were more likely to view weaknesses as changeable. Second, self-compassion was positively associated with the motivation to avoid repeating a moral transgression. It was also associated with higher scores on subsequent tests by studying for longer periods of time after initial test failure. Third, self-comparison was linked to greater preference to socially interact with individuals who had overcome a similar weakness to their own rather than individuals who had not addressed their weakness i.e. upward social comparison. Last, self-compassion was associated with higher motivation to change weaknesses. It was concluded that self-compassion may in fact be an adaptive response to failure in light of self-improvement motivation (Breanes \& Chen, 2012). Sheperd and Cardon (2009) studied the role of self-compassion in project failure experiences in entrepreneurial organizations. They found that self-compassion aids an individual to learn from a failure experience rather than engage in ego defense mechanisms that obstruct learning. This suggests that self-compassion would do the opposite of promoting passivity (Sheperd \& Cordon, 2009).

However, given the belief that seems to exist among athletes that self-compassion may promote acceptance of mediocrity (Ferguson et al., 2014; Reis et al., 2015; Sutherland et al., 
2014), Reis et al. (2015) suggested that interventions and studies designed to promote selfcompassion should make special consideration for athlete's fear of self-compassion as this may lead them to resist the benefits of adopting a self-compassionate mindset. Rather, future studies that attempt to assess levels of self-compassion or induce self-compassion in athletes may want to provide athletes with a rationale for the benefits of self-compassion on well-being and the role that self-compassion plays in aiding an individual to learn from a failure experience rather than engage in ego defense mechanisms that obstruct learning. This may reduce reluctance on the athlete's part to reveal their own levels of self-compassion.

\section{Intermittent Conclusions}

Self-compassion is composed of three distinct concepts: self-kindness in times of perceived inadequacy, common humanity, and balanced awareness of emotions (Neff, 2003a; Neff, 2003b). Self-compassion is associated with well-being, reduced anxiety and positive psychological functioning (Neely et al., 2009; Neff, 2003a; Neff et al., 2007a). Within the sport and exercise psychology context, self-compassion has been found to be negatively correlated with shame-proneness, social physique anxiety, fear of failure and fear of negative evaluation in female athletes (Mosewich et al., 2011). Self-compassion has also been associated with healthier responses in female athletes to emotionally difficult hypothetical and recalled sport situations as well as lower levels of self-criticism (Mosewich et al, 2013; Reis et al., 2015). Findings from these few studies suggest that self-compassion may be a potential resource for female athletes. Direct research into the role of self-compassion for female athletes is warranted and will be addressed by the proposed study. Furthermore, research into the role of self-compassion in male athletes has been particularly sparse and will be further explored in the proposed study. Selfcompassion may be a particularly appropriate psychological construct to explore in athletes 
because it is modifiable both through short-term interventions such as self-compassion writing inductions (Leary et al., 2007) and longer-term interventions such as MSBR (Chiesa \& Serretti, 2009), CMT (Gilbert \& Proctor, 2006) and LKM (Grossman \& Hinton, 2011).

\section{SELF-COMPASSION, STRESS AND INJURY IN SPORT}

\section{Self-Compassion as a Moderator of the Relationship between Stress and Injury in Sport}

Effective coping skills are invaluable for athletes given the amount of stress they face. Mosewich, Crocker and Kowalski (2014) studied setbacks and coping in high-performance female athletes. Findings indicated that athletes most commonly managed setbacks through the use of striving for balance and reducing self-criticism. This is particularly interesting given that self-compassion has been found to be helpful in reducing self-criticism in female athletes (Mosewich et al., 2013) as well as promoting balanced awareness of emotions (Leary et al., 2007). The component of self-compassion that involves balanced awareness of one's emotions may help individuals face uncomfortable experiences and painful thoughts without resorting to avoidance strategies (Allen \& Leary, 2010; Neff, Kirkpatrick \& Rude, 2007b). Although selfcompassion was not directly measured, Mosewichet al. (2014) suggested that self-compassion may be particularly useful in managing issues that athletes find challenging and stressful such as poor performance, performance plateau and injury.

Given the consensus that stress management techniques can significantly reduce the prevalence of injury in collegiate athletes ((Edvarsson, Ivarsson \& Johnson, 2012; Ivarsson et al., 2015; Johnson, Ekengren \& Andersen, 2005; Perna et al., 2003), it is justifiable to further explore other avenues for coping with stress. Self-compassion enhances adaptive coping, positive psychological functioning and well-being in the face of stress (Neely et al., 2009; Neff, 2003b; Neff, Kirkpatrick \& Rude, 2007b; Allen \& Leary, 2010) and contributes to the appraisal 
process by changing the experience of self-evaluative appraisals (Neff, 2003a; Neff et al., 2007a; Mosewich et al., 2011). Importantly, self-compassion is a form of emotion-focused coping (Neff, 2003a) and allows for accurate self-appraisals to be made while maintaining balanced awareness of emotions (Allen \& Leary, 2010; Leary et al., 2007). Therefore, if self-compassion could be used to reduce stress i.e. as a stress management or coping tool, then it could be successfully implemented as a psychological intervention for athletic injury.

In relation to Williams and Andersen's (1998) stress and injury model, self-compassion may serve as one of the moderators of the relationship between stress and athletic injury. Williams and Anderson (1998) and Petrie (1993) suggested that psychosocial variables do not play a direct role in vulnerability to injury (i.e. they are not independent variables) and are instead moderators so it follows that self-compassion may hold the same effect. In the stress and injury model, self-compassion could be considered a coping resource. Importantly, within this proposed version of the stress and injury model, self-compassion would have an influence on the coping style that the athlete employs. This is consistent with research that suggests that selfcompassion is an emotional approach style of coping (Neff, 2003a) that uses positive reinterpretation of negative events to deal with the emotional distress associated with a stressful situation (Neff, Hsieh \& Dejitterat, 2005). Focusing on emotions may allow the individual (in this case the athlete) to re-appraise the stressor and come to a healthier attribution of the event (Stanton et al., 2000). Furthermore, self-compassion has been positively associated with the emotion-focused coping strategies of growth and acceptance as well as positive re-interpretation while it was negatively associated with the emotion-focused strategy of venting of emotions (Neff, Hsieh \& Dejitterat, 2005). Additionally, self-compassion is also negatively correlated with 
avoidance coping (Allen \& Leary, 2010); specifically, the avoidance coping strategies of denial and mental disengagement (Neff, Hsieh \& Dejitterat, 2005).

The literature outside of the athletic context therefore suggests that there is a strong argument for the use of self-compassion as a coping resource for stress in athletes. This has yet to be studied in a formal manner and has yet to be studied within the framework of Williams and Andersen's stress-injury model. The key concept that will connect self-compassion, stress and injury in Williams and Andersen's (1998) stress and injury model is appraisal. Self-compassion has an impact on coping style through its effects on appraisal of stress. Self-compassionate individuals are better able to make accurate self-appraisals and accept responsibility for the role they play in the unfolding of negative events while maintaining balanced emotional awareness (Allen \& Leary, 2010; Leary et al., 2007). In this way, self-compassion changes appraisal of stress as well as coping style, which impact the stress response and in turn contribute to the link between stress and injury.

Proposed Model:

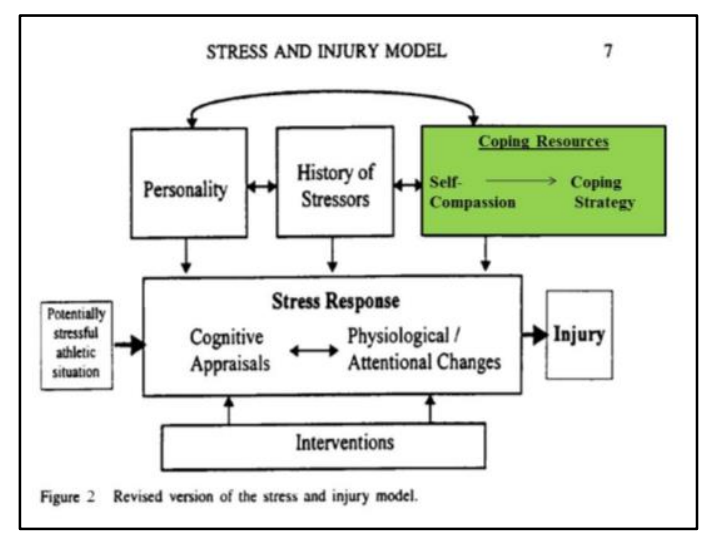




\section{Appendix C: Basic Demographics Sheet}

1. What is your name?

2. What is your gender?
Male
Female

3. What is your age?

4. What is your ethnicity?

American Indian/Alaskan Native

Asian or Asian American

Black or African American

Hispanic or Latino

White or Caucasian

Other (please specify)

5. What is your highest level of education?

High School

Some College

College Degree

Some Graduate School

Graduate Degree

Doctoral Degree

Other

6. What is your year in school?

Freshman _ Sophomore _ Junior _ Senior _ G Graduate student

7. What is your playing status on the team?

_ Starter _ Second string _ Third string

8. How many years have you been playing this sport?

9. Have you sustained any major athletic injuries during the past year?

Yes_ No

10. If so, state the severity of the injury and the type of injury e.g. ankle sprain.
a. Severity:
Type:
b. Severity:
Type:
c. Severity:
Type:

** severity of injury refers to the length of time that the injury kept you out of practice and competition: a) minor (1-7 days), b) moderate (1 week to 1 month) and c) major injuries (>1 month) 


\section{Appendix D: Life Events Survey for Collegiate Athletes (LESCA)}

Listed below are 69 events which sometimes occur in the lives of collegiate athletes. These events often produce change within an individual's life that require some adjustment by the individual. For each event that you have experiences within the last year (12 months):

1. Place a check under the column 0 months to 1 year to indicate that you have experienced the event within the last year. If you have not experienced an event within the last year, leave that item blank.

2. Then, rate from -4 (extremely negative) to +4 (extremely positive) what kind of an effect it had on your life when the event occurred. For those events that have happened more than once in the last year, indicate the average effect across all occurrences.

$\begin{array}{ll}-4 & \text { Extremely Negative } \\ -3 & \text { Negative } \\ -2 & \text { Moderately Negative } \\ -1 & \text { Somewhat Negative } \\ +1 & \text { Somewhat positive } \\ +2 & \text { Moderately Positive } \\ +3 & \text { Positive } \\ +4 & \text { Extremely Positive }\end{array}$


(0 months to one year)

RATING OF EFFECT

1. Marriage

2. Death of a mate (boyfriend, girlfriend, spouse, significant other)

3. Major change in sleeping habits (increase or decrease in amount of sleep)

4. Death of a close family member(s)
a. father
b. mother
c. brother
d. sister
e. grandfather
f. grandmother
g. other

5. Major change in eating habits (increase or decrease in food intake)

6. Death of close friend(s)

7. Outstanding personal achievement

8. MALE: Mate pregnant

9. FEMALE: Becoming pregnant

10. Sexual difficulties

11. Being fired from job

12. Being apart from mate (boy/girlfriend, spouse... etc.) - due to sport

\begin{tabular}{|c|c|c|c|c|c|c|c|}
\hline-4 & -3 & -2 & -1 & +1 & +2 & +3 & +4 \\
\hline-4 & -3 & -2 & -1 & +1 & +2 & +3 & +4 \\
\hline-4 & -3 & -2 & -1 & +1 & +2 & +3 & +4 \\
\hline-4 & -3 & -2 & -1 & +1 & +2 & +3 & +4 \\
\hline-4 & -3 & -2 & -1 & +1 & +2 & +3 & +4 \\
\hline-4 & -3 & -2 & -1 & +1 & +2 & +3 & +4 \\
\hline-4 & -3 & -2 & -1 & +1 & +2 & +3 & +4 \\
\hline-4 & -3 & -2 & -1 & +1 & +2 & +3 & +4 \\
\hline-4 & -3 & -2 & -1 & +1 & +2 & +3 & +4 \\
\hline-4 & -3 & -2 & -1 & +1 & +2 & +3 & +4 \\
\hline-4 & -3 & -2 & -1 & +1 & +2 & +3 & +4 \\
\hline-4 & -3 & -2 & -1 & +1 & +2 & +3 & +4 \\
\hline-4 & -3 & -2 & -1 & +1 & +2 & +3 & +4 \\
\hline-4 & -3 & -2 & -1 & +1 & +2 & +3 & +4 \\
\hline-4 & -3 & -2 & -1 & +1 & +2 & +3 & +4 \\
\hline-4 & -3 & -2 & -1 & +1 & +2 & +3 & +4 \\
\hline-4 & -3 & -2 & -1 & +1 & +2 & +3 & +4 \\
\hline-4 & -3 & -2 & -1 & +1 & +2 & +3 & +4 \\
\hline-4 & -3 & -2 & -1 & +1 & +2 & +3 & +4 \\
\hline
\end{tabular}


13. Serious illness or injury of close family

$\left[\begin{array}{llllllll}-4 & -3 & -2 & -1 & +1 & +2 & +3 & +4\end{array}\right.$
member(s)
a. father
b. mother
c. brother
d. sister
e. grandfather
f. grandmother
g. other

$\begin{array}{lrrrrrrrr}- & -4 & -3 & -2 & -1 & +1 & +2 & +3 & +4 \\ - & -4 & -3 & -2 & -1 & +1 & +2 & +3 & +4 \\ - & -4 & -3 & -2 & -1 & +1 & +2 & +3 & +4 \\ - & -4 & -3 & -2 & -1 & +1 & +2 & +3 & +4 \\ - & -4 & -3 & -2 & -1 & +1 & +2 & +3 & +4 \\ - & -4 & -3 & -2 & -1 & +1 & +2 & +3 & +4 \\ - & -4 & -3 & -2 & -1 & +1 & +2 & +3 & +4 \\ - & -4 & -3 & -2 & -1 & +1 & +2 & +3 & +4\end{array}$
14. Major change in the number (more or less) of $\begin{array}{llllllll}-4 & -3 & -2 & -1 & +1 & +2 & +3 & +4\end{array}$ arguments with mate

15. Major personal injury or illness

16. Major change in frequency (increased or

$\begin{array}{lllllllll}-4 & -3 & -2 & -1 & +1 & +2 & +3 & +4 \\ - & -4 & -3 & -2 & -1 & +1 & +2 & +3 & +4\end{array}$ decreased) of social activities due to participation in sport

17. Serious injury or illness of close friend

18. Breaking up with mate (boy/girlfriend etc.)

$\begin{array}{lrrrrrrrr}- & -4 & -3 & -2 & -1 & +1 & +2 & +3 & +4 \\ - & -4 & -3 & -2 & -1 & +1 & +2 & +3 & +4 \\ & -4 & -3 & -2 & -1 & +1 & +2 & +3 & +4\end{array}$

19. Beginning a new school experience (beginning college, transferring colleges etc.)

20. Engagement

$\begin{array}{lllllllll}- & -4 & -3 & -2 & -1 & +1 & +2 & +3 & +4 \\ - & -4 & -3 & -2 & -1 & +1 & +2 & +3 & +4 \\ - & -4 & -3 & -2 & -1 & +1 & +2 & +3 & +4 \\ - & -4 & -3 & -2 & -1 & +1 & +2 & +3 & +4 \\ - & -4 & -3 & -2 & -1 & +1 & +2 & +3 & +4\end{array}$
(better or worse)

25. Failing a course

$\begin{array}{lllllllll}- & -4 & -3 & -2 & -1 & +1 & +2 & +3 & +4 \\ - & -4 & -3 & -2 & -1 & +1 & +2 & +3 & +4\end{array}$ of practice/training (better or worse) 
27. Financial problems concerning school

28. Major change in relationship with family member(s) (better or worse)

29. Conflict with roommate

30. Male: Mate having an abortion

31. Female: Having an abortion

32. Major change in the amount (more or less) of academic activity (homework, class time etc.)

33. Pressure to gain/lose weight- due to participation in sport

34. Discrimination from teammates/coaches

35. Major change in relationship(s) with teammate(s) (better or worse)

36. Suspended from team for nonacademic reasons

37. Trouble with academic counselor

38. Major change in use of alcohol/drugs (increased or decreased)

39. Beginning sexual activity

40. Major change in relationship(s) with friend(s) (better or worse)

41. recovery from illness/injury/operation 42. Major change in level of athletic performance in actual competition (better or worse) $\left[\begin{array}{llllllll}-4 & -3 & -2 & -1 & +1 & +2 & +3 & +4\end{array}\right.$

$\left[\begin{array}{llllllll}-4 & -3 & -2 & -1 & +1 & +2 & +3 & +4\end{array}\right.$

$\left[\begin{array}{lllllllll}-4 & -3 & -2 & -1 & +1 & +2 & +3 & +4\end{array}\right.$

$\left[\begin{array}{llllllll}-4 & -3 & -2 & -1 & +1 & +2 & +3 & +4\end{array}\right.$

$\left[\begin{array}{llllllll}-4 & -3 & -2 & -1 & +1 & +2 & +3 & +4\end{array}\right.$

$\begin{array}{llllllll}-4 & -3 & -2 & -1 & +1 & +2 & +3 & +4\end{array}$

$\begin{array}{lllllllll}-4 & -3 & -2 & -1 & +1 & +2 & +3 & +4\end{array}$

$\left[\begin{array}{llllllll}-4 & -3 & -2 & -1 & +1 & +2 & +3 & +4\end{array}\right.$

$\left[\begin{array}{lllllllll}-4 & -3 & -2 & -1 & +1 & +2 & +3 & +4\end{array}\right.$

$\left[\begin{array}{lllllllll}-4 & -3 & -2 & -1 & +1 & +2 & +3 & +4\end{array}\right.$

$\begin{array}{lllllllll}- & -4 & -3 & -2 & -1 & +1 & +2 & +3 & +4\end{array}$

$\left[\begin{array}{llllllll}-4 & -3 & -2 & -1 & +1 & +2 & +3 & +4\end{array}\right.$

$\begin{array}{lllllllll}- & -4 & -3 & -2 & -1 & +1 & +2 & +3 & +4\end{array}$

$\begin{array}{llllllll}-4 & -3 & -2 & -1 & +1 & +2 & +3 & +4\end{array}$

$\begin{array}{lllllllll}-4 & -3 & -2 & -1 & +1 & +2 & +3 & +4\end{array}$

$\begin{array}{lllllllll}- & -4 & -3 & -2 & -1 & +1 & +2 & +3 & +4\end{array}$ 
43. Divorce or separation of your parents

44. Major change in level of responsibility on team (increased or decreased)

45. receiving an athletic scholarship

46. Not attaining personal goals in sport

47. Major change in playing status on team

48. injury to teammates

49. Being absent from school (classes) because of participation in sport

50. Troubles with athletic association and/or athletic director

51. Difficulties with trainer/physician

52. Major change in playing time (playing more or less) - due to injury)

53. Major errors/mistakes in actual competition

54. Losing your athletic scholarship

55. No recognition/praise of accomplishments from coaching staff

56. Pressure from family to perform well

57. Loss of confidence due to injury

58. Unable to find a job

59. Change in coaching staff

60. Female: menstrual period/PMS

61. Major change in level of academic

$\begin{array}{ccccccccc}- & -4 & -3 & -2 & -1 & +1 & +2 & +3 & +4 \\ - & -4 & -3 & -2 & -1 & +1 & +2 & +3 & +4\end{array}$

$\left[\begin{array}{llllllll}-4 & -3 & -2 & -1 & +1 & +2 & +3 & +4\end{array}\right.$

$\begin{array}{llllllll}-4 & -3 & -2 & -1 & +1 & +2 & +3 & +4\end{array}$

$\begin{array}{lllllllll}-4 & -3 & -2 & -1 & +1 & +2 & +3 & +4\end{array}$

$\begin{array}{llllllll}-4 & -3 & -2 & -1 & +1 & +2 & +3 & +4\end{array}$

$\begin{array}{llllllll}-4 & -3 & -2 & -1 & +1 & +2 & +3 & +4\end{array}$

$\begin{array}{lllllllll}-4 & -3 & -2 & -1 & +1 & +2 & +3 & +4\end{array}$

$\begin{array}{lllllllll}-4 & -3 & -2 & -1 & +1 & +2 & +3 & +4\end{array}$

$\begin{array}{llllllll}-4 & -3 & -2 & -1 & +1 & +2 & +3 & +4\end{array}$

$\left[\begin{array}{llllllll}-4 & -3 & -2 & -1 & +1 & +2 & +3 & +4\end{array}\right.$

$\left[\begin{array}{lllllllll}-4 & -3 & -2 & -1 & +1 & +2 & +3 & +4\end{array}\right.$

$\left[\begin{array}{llllllll}-4 & -3 & -2 & -1 & +1 & +2 & +3 & +4\end{array}\right.$

$\left[\begin{array}{lllllllll}-4 & -3 & -2 & -1 & +1 & +2 & +3 & +4\end{array}\right.$

$\left[\begin{array}{lllllllll}-4 & -3 & -2 & -1 & +1 & +2 & +3 & +4\end{array}\right.$

$\left[\begin{array}{lllllllll}-4 & -3 & -2 & -1 & +1 & +2 & +3 & +4\end{array}\right.$

$\begin{array}{lllllllll}-4 & -3 & -2 & -1 & +1 & +2 & +3 & +4\end{array}$

$\begin{array}{lllllllll}- & -4 & -3 & -2 & -1 & +1 & +2 & +3 & +4\end{array}$

$\begin{array}{llllllll}-4 & -3 & -2 & -1 & +1 & +2 & +3 & +4\end{array}$ 
performance (doing better or worse)

62. Making career decisions (applying to graduate school, interviewing for jobs, etc.)

63. Being cut/dropped from the team

$\begin{array}{ccccccccc}-4 & -3 & -2 & -1 & +1 & +2 & +3 & +4 \\ & -4 & -3 & -2 & -1 & +1 & +2 & +3 & +4 \\ - & -4 & -3 & -2 & -1 & +1 & +2 & +3 & +4 \\ & -4 & -3 & -2 & -1 & +1 & +2 & +3 & +4\end{array}$

66. Major change in family finances (increased or decreased)

67. Major change in attitude toward sport ( $\left[\begin{array}{llllllll}-4 & -3 & -2 & -1 & +1 & +2 & +3 & +4\end{array}\right.$ like/enjoy more or less)

68. Victim of harassment/abuse (sexual, emotional, physical)

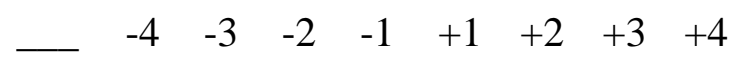

69. Victim of personal attack (rape, robbery, $\left[\begin{array}{llllllll}-4 & -3 & -2 & -1 & +1 & +2 & +3 & +4\end{array}\right.$ assault etc.)

Other events might have occurred to you in the past year (and affected you in a positive or negative manner) but were not included in this list. If there were such events, please list them below.

70.

71.

72.

73.

74. 


\section{Appendix E: Sport Anxiety Scale (SAS)}

Instructions: The following are statements that athletes have used to describe their thoughts and feelings while participating in their sport. Please read each statement carefully, and then rate the extent to which they relate to your own experiences when playing sport.

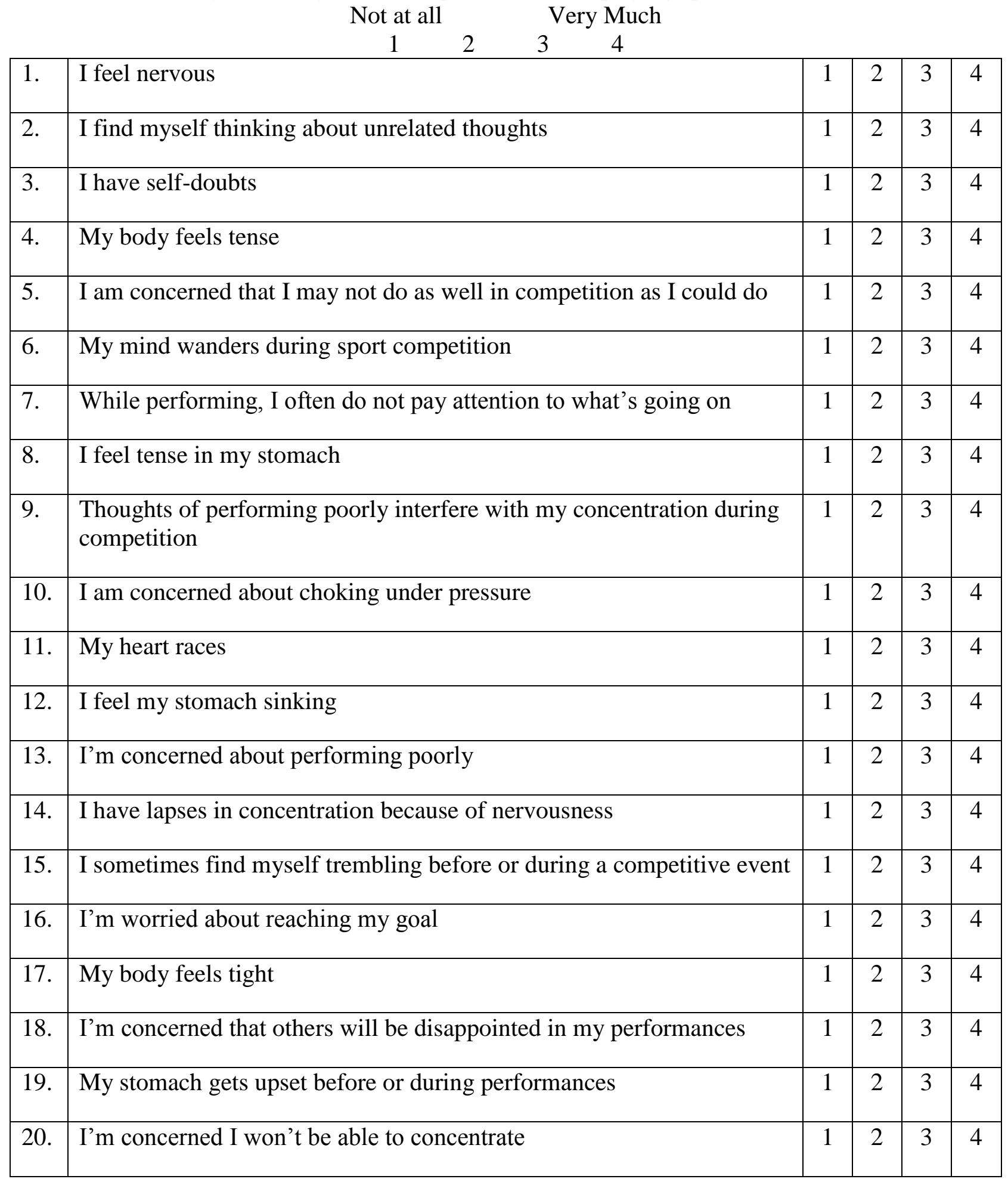




\begin{tabular}{|l|l|l|l|l|l|}
\hline 21. & My heart pounds before competition & 1 & 2 & 3 & 4 \\
\hline
\end{tabular}

\section{Appendix F: Self-Compassion Scale- Short Form (SCS-SF)}

Please read each statement carefully before answering. To the left of each item, indicate how often you behave in the stated manner, using the following scale:

$\begin{array}{ccccc}\text { Almost never } & & \text { Almost always } \\ 1 & 2 & 3 & 4 & 5\end{array}$

1. When I fail at something important to me I become consumed by feelings of inadequacy. like.

2. I try to be understanding and patient towards those aspects of my personality I don't

3. When something painful happens I try to take a balanced view of the situation. I am.

4. When I'm feeling down, I tend to feel like most other people are probably happier than

5. I try to see my failings as part of the human condition. need.

6. When I'm going through a very hard time, I give myself the caring and tenderness I

7. When something upsets me I try to keep my emotions in balance.

8. When I fail at something that's important to me, I tend to feel alone in my failure

9. When I'm feeling down I tend to obsess and fixate on everything that's wrong.

10. When I feel inadequate in some way, I try to remind myself that feelings of inadequacy are shared by most people.

11. I'm disapproving and judgmental about my own flaws and inadequacies.

12. I'm intolerant and impatient towards those aspects of my personality I don't like. 


\section{Appendix G: Brief COPE}

These items deal with ways you've been coping with stress in your life. There are many ways to try to deal with problems. These items ask what you've been doing to cope with this one. Obviously, different people deal with things in different ways, but I'm interested in how you've tried to deal with it. Each item says something about a particular way of coping. I want to know to what extent you've been doing what the item says. How much or how frequently. Don't answer on the basis of whether it seems to be working or not - Just whether or not you're doing it. Use these response choices. Try to rate each item separately in your mind from the other. Make your answers as true FOR YOU as you can.

$1=\mathrm{I}$ haven't been doing this at all

$2=$ I've been doing this a little bit

$3=$ I've been doing this a medium amount

$4=$ I've been doing this a lot

1. I've been turning to work or other activities to take my mind off things.

2. I've been concentrating my efforts on doing something about the situation I'm in.

3. I've been saying to myself "this isn't real".

4. I've been using alcohol or other drugs to make myself feel better.

5. I've been getting emotional support from others.

6. I've been giving up trying to deal with it.

7. I've been taking action to try to make the situation better.

8. I've been refusing to believe that it has happened.

9. I've been saying things to let my unpleasant feelings escape.

10. I've been getting help and advice from other people.

11. I've been using alcohol or other drugs to help me get through it.

12. I've been trying to see it in a different light, to make it seem more positive.

13. I've been criticizing myself.

14. I've been trying to come up with a strategy about what to do.

$\begin{array}{llll}1 & 2 & 3 & 4 \\ 1 & 2 & 3 & 4 \\ 1 & 2 & 3 & 4 \\ 1 & 2 & 3 & 4 \\ 1 & 2 & 3 & 4 \\ 1 & 2 & 3 & 4 \\ 1 & 2 & 3 & 4 \\ 1 & 2 & 3 & 4 \\ 1 & 2 & 3 & 4 \\ 1 & 2 & 3 & 4 \\ 1 & 2 & 3 & 4 \\ 1 & 2 & 3 & 4 \\ 1 & 2 & 3 & 4 \\ 1 & 2 & 3 & 4\end{array}$


15. I've been getting comfort and understanding from someone. $\quad \begin{array}{lllll}1 & 2 & 3 & 4\end{array}$

16. I've been giving up attempting to cope. $\quad \begin{array}{lllll}1 & 2 & 3 & 4\end{array}$

17. I've been joking for something good in what is happening. $\quad \begin{array}{lllll}1 & 2 & 3 & 4\end{array}$

18. I've been making jokes about it. $\quad \begin{array}{llll}1 & 2 & 3 & 4\end{array}$

19. I've been doing something to think about it less, such as going to $\quad \begin{array}{lllll}1 & 2 & 3 & 4\end{array}$ movies, watching TV, reading, daydreaming, sleeping, or shopping.

20. I've been accepting the reality of the fact that it has happened. $\quad \begin{array}{lllll}1 & 2 & 3 & 4\end{array}$

21. I've been expressing my negative feelings. $\quad \begin{array}{lllll}1 & 2 & 3 & 4\end{array}$

22. I've been trying to find comfort in my religion or spiritual beliefs. $\begin{array}{lllll}1 & 2 & 3 & 4\end{array}$

23. I've been trying to get advice or help from other people about what $\begin{array}{lllll}1 & 2 & 3 & 4\end{array}$ to do.

24. I've been learning to live with it. $\quad \begin{array}{llll}1 & 2 & 3 & 4\end{array}$

$\begin{array}{lllll}\text { 25. I've been thinking hard about what steps to take. } & 1 & 2 & 3 & 4\end{array}$

26. I've been blaming myself for things that happened. $\quad \begin{array}{lllll}1 & 2 & 3 & 4\end{array}$

27. I've been praying or meditating. $\quad \begin{array}{lllll}1 & 2 & 3 & 4\end{array}$

28. I've been making fun of the situation. $\quad \begin{array}{lllll}1 & 2 & 3 & 4\end{array}$ 


\section{Appendix H: Athletic Coping Skills Inventory}

Instructions: The following are statements that athletes have used to describe their experiences. Please read each statement carefully, and then recall as accurately as possible how often you experience the same thing. There are no right or wrong answers. Do not spend too much time on any one statement.

Please circle how often you have these experiences when playing sports.

1 On a daily or weekly basis, I set very specific goals for myself that guide what I Almost do. never Sometimes Often Almost

Almost

Sometimes Often

Almost

I get the most out of my talent and skill. never Sometimes always

3 When a coach or manager tells me how to correct a mistake I've made, I tend to take it personally and feel upset.

Almost Almost never Sometimes Often always

4 When I'm playing sports, I can focus my Almost Almost attention and block out distractions. never

Sometimes Often always

5 I remain positive and enthusiastic during competition, no matter how badly things are going.

Almost never

Almost never because I think more clearly.

7 I worry quite a bit about what others think of my performance.

8 I tend to do lots of planning about how to reach my goals.
I feel confident that I will play well.
Almost never

Sometimes Often

Almost

Almost

Sometimes

Often

Almost never

Almost never always always Almost Sometimes Often always
Almost always

Almost always 
10 When a coach or manager criticizes me, I Almost

never

Sometimes Often always

Almost become upset rather than feel helped.

11 It is easy for me to keep distracting

thoughts from interfering with something I Almost

Sometimes Often always

12 I put a lot of pressure on myself by never

Almost

Almost worrying about how I will perform.

never

Sometimes Often

always

13 I set my own performance goals for each

Almost practice.

never

Sometimes Often

Almost

always

14 I don't have to be pushed to practice or

Almost

Almost play hard; I give $100 \%$. never

Sometimes Often

always

15 If a coach criticizes or yells at me, I correct Almost Sometimes Often

Almost the mistake without getting upset about it. never

Almost never

Sometimes

Often

Almost very well.

Almost never

Sometimes

Often

Almost to keep calm, and this works for me.

Almost never

Sometimes

Often

Almost the more I enjoy it.

Almost never

Sometimes

Often

Almost mistakes or failing to come through.

Almost never

Sometimes Often

Almost my head long before the game begins.

21 When I feel myself getting too tense, I can Almost never

Sometimes Often

Almost quickly relax my body and calm myself.

Almost

Almost never

Sometimes

Often always 
23 I think about and imagine what will

Almost happen if I fail or screw up.

24 I maintain emotional control regardless of how things are going for me.

25 It is easy for me to direct my attention and focus on a single object or person.

26 When I fail to reach my goals, it makes me try even harder.

27 I improve my skills by listening carefully to advice and instruction from coaches and managers.

28 I make fewer mistakes when the pressure is on because I concentrate better. never

Almost never

Almost never

Almost never

Almost never

Almost never
Sometimes Often

Almost always

Almost Sometimes Often always

Sometimes Often

Almost always

Almost Sometimes Often always

Almost

Sometimes Often always

Almost Sometimes Often always 


\section{Appendix I: Order of Survey Packets}

\section{Survey Packet 1}

1. Basic Demographic sheet

2. Life Events Survey for Collegiate Athletes (LESCA)

3. Self-Compassion Scale Short Form (SCS-SF)

4. Brief COPE

5. Sport Anxiety Scale (SAS)

6. Athletic Coping Skills Inventory (ACSI-28)

\section{Survey Packet 2}

1. Basic Demographic sheet

2. Athletic Coping Skills Inventory (ACSI-28)

3. Sport Anxiety Scale (SAS)

4. Life Events Survey for Collegiate Athletes (LESCA)

5. Brief COPE

6. Self-Compassion Scale Short Form (SCS-SF)

\section{Survey Packet 3}

1. Basic Demographic sheet

2. Brief COPE

3. Sport Anxiety Scale (SAS)

4. Athletic Coping Skills Inventory (ACSI-28)

5. Self-Compassion Scale Short Form (SCS-SF)

6. Life Events Survey for Collegiate Athletes (LESCA) 